\title{
LÉVY PROCESSES WITH ADAPTABLE EXPONENT
}

\author{
R. BEKKER, * VU University Amsterdam \\ O. J. BOXMA,** EURANDOM and Eindhoven University of Technology \\ J. A. C. RESING, ${ }^{* *}$ Eindhoven University of Technology
}

\begin{abstract}
In this paper we consider Lévy processes without negative jumps, reflected at the origin. Feedback information about the level of the Lévy process ('workload level') may lead to adaptation of the Lévy exponent. Examples of such models are queueing models in which the service speed or customer arrival rate changes depending on the workload level, and dam models in which the release rate depends on the buffer content. We first consider a class of models where information about the workload level is continuously available. In particular, we consider dam processes with a two-step release rule and M/G/1 queues in which the arrival rate, service speed, and/or jump size distribution may be adapted depending on whether the workload is above or below some level $K$. Secondly, we consider a class of models in which the workload can only be observed at Poisson instants. At these Poisson instants, the Lévy exponent may be adapted based on the amount of work present. For both classes of models, we determine the steady-state workload distribution.
\end{abstract}

Keywords: Reflected Lévy process; adaptable exponent; workload distribution; scale function; Laplace inversion; M/G/1 queue; storage process

2000 Mathematics Subject Classification: Primary 60K25; 60J30

\section{Introduction}

The basic process under consideration in this paper is a spectrally one-sided Lévy process $\{X(t), t \geq 0\}[6]$, i.e. a Lévy process which has either no positive or no negative jumps. Such processes are often studied in the applied probability literature, with applications to, for example, queues, dams, storage processes, and finance. It is well known that we can write

$$
\mathrm{E}\left[\mathrm{e}^{-\omega X(t)}\right]=\mathrm{e}^{t \phi(\omega)}
$$

$\phi(\omega)$ is called the Lévy exponent. In particular, we will mainly consider Lévy processes without negative jumps, reflected at the origin. The key feature of the paper is that the Lévy exponent may change, depending on the level of the Lévy process ('workload level'). For example, consider the classical M/G/1 queue with service speed $r$, arrival rate $\lambda$, and service requirement distribution $B(\cdot)$ with Laplace-Stieltjes transform (LST) $\beta(\cdot)$. The input process to the queue is a compound Poisson process, which is a special case of a Lévy process, having Lévy exponent $-\lambda(1-\beta(\omega))$. Subtracting a deterministic drift of rate $r$, to take the service capacity into account, yields a Lévy process with Lévy exponent $r \omega-\lambda(1-\beta(\omega))$. Information about the

Received 18 July 2007; revision received 8 December 2008.

* Postal address: Department of Mathematics, VU University Amsterdam, De Boelelaan 1081a, 1081 HV Amsterdam, The Netherlands. Email address: rbekker@few.vu.nl

** Postal address: Department of Mathematics and Computer Science, Eindhoven University of Technology, PO Box 513, 5600 MB Eindhoven, The Netherlands. 
workload level may lead one to change the service speed $r$, the arrival rate $\lambda$, and/or the service requirement LST $\beta(\cdot)$, and, hence, the Lévy exponent.

There is a large literature about queueing models in which the server may work at different speeds, depending on either the number of customers in the system or the amount of work in the system. Models with service speed depending on queue length or workload arise naturally as representation of congestion phenomena in, for example, manufacturing and healthcare processes. Another bulk of papers is devoted to the (related) case in which the arrival rate of customers depends on the state of the system. For example, feedback information signals are being used in various communication systems to regulate the offered traffic volume in accordance with the actual level of congestion. A prime example is the transmission control protocol in the Internet. The case of service requirement distributions depending on the workload level has received much less attention; see, e.g. [9] and [31]. We refer the reader to [16] for an extensive survey, with 277 references, on queueing models with state-dependent parameters.

Another application area of Lévy processes without negative jumps and state-dependent exponents concerns the analysis of dam models, in which the level of the buffer content increases (or decreases) gradually during stochastic amounts of time. Dams form a historically important area of systems with state-dependent rates. The input process of a large class of dam processes studied in the literature (see [11], [15], [30, Chapter 3], or [32]) is a nondecreasing Lévy process. Information about the level of the Lévy process (which we call the workload) may lead to adaptation of the release rate or, more generally, the Lévy exponent.

Lévy processes without positive jumps and with state-dependent exponents are also important in finance, where they are sometimes called refracted Lévy processes (see [23]). In this area, contrary to the queueing and dam applications, the processes are typically not reflected at 0 and we are interested in first-exit problems instead of steady-state distributions. A typical class of models falling into this framework are surplus processes in which dividends are paid according to a generalized barrier strategy (also called a threshold strategy). According to such a strategy, dividends are paid at a constant rate whenever the surplus is above the threshold and no dividends are paid whenever the surplus is below the threshold; see [18], [19], and [26].

In the present study we restrict ourselves to two classes of models in which the determining factor is whether the workload is above or below a certain level $K$. First we briefly consider a class of models where information about the level of congestion (workload) is continuously available. In particular, we consider dam processes with a two-step release rule (corresponding to the service speed) and M/G/1 queues with adaptable arrival rate, service speed, and/or service requirement distribution, depending on whether the workload is above or below level $K$. The drawback of these models is that frequent changes in the adaptable parameters are not excluded. In the second class of models, the workload can only be observed at Poisson instants, and then the adaptable parameters can be changed based on the amount of work present. This is the main model class of the paper and we refer to it as the Poisson observer model.

The main goal of the paper is to derive the steady-state distribution of the workload of the reflected Lévy process. We develop a solution procedure that can be used to determine such steady-state behavior for a large class of reflected Lévy processes with two Lévy exponents. The machinery used includes Laplace transforms (building upon a technique developed in [17] (see also [4])), martingales, and various properties of Lévy processes.

This paper is organized as follows. Section 2 contains descriptions of the dam (and M/G/1 queue) and the model with a Poisson observer, and some preliminary results on reflected Lévy processes without negative jumps. In Section 3 we give an outline of a five-step procedure to 
determine the steady-state behavior of various queueing systems with two service rates or, more generally, reflected Lévy processes with two Lévy exponents. In Sections 4-6 we apply this procedure to several models. In Section 4 we present the steady-state workload analysis of the dam and M/G/1 system that has different arrival rates, service speeds, and service requirements when the workload is above or below $K$. As an example, we derive the steady-state workload distribution in a dam with two release rates (service speeds) in Subsection 4.1. In Subsection 4.2 we consider the general M/G/1 queue where, in addition to the service speed, the arrival rate and service requirements can also be adapted based on the workload. The model of a reflected Lévy process with a Poisson observer is analyzed in Sections 5 and 6. In this analysis a key role is played by so-called alternating Lévy processes reflected at the origin. These processes are studied in Appendix A.

\section{Models and preliminaries}

In this section we introduce the two models considered in the paper. Moreover, we give some preliminary results on reflected Lévy processes without negative jumps.

First some notation. For some process, denote the value of that process at time $t$ by $X^{(t)}$ and let $X$ correspond to its steady-state version (if it exists). We specifically consider Lévy processes without negative jumps $\left\{X_{i}^{(t)}, t \geq 0\right\}, i=1,2$. For convenience, we exclude cases in which the processes $\left\{X_{i}^{(t)}, t \geq 0\right\}, i=1,2$, have monotone paths. (A similar approach can however be applied to the case where $X_{1}^{(t)}$ is a subordinator or $X_{2}^{(t)}$ is the negative of a subordinator.) Denote by $\phi_{i}(\cdot)$ the Lévy exponent of $\left\{X_{i}^{(t)}, t \geq 0\right\}$, i.e.

$$
\mathrm{E}\left[\exp \left\{-\omega X_{i}^{(t)}\right\}\right]=\exp \left\{t \phi_{i}(\omega)\right\} .
$$

Let $Z_{i}^{(t)}=Z_{i}^{(0)}+X_{i}^{(t)}+L_{i}^{(t)}, t \geq 0$, starting at $Z_{i}^{(0)} \geq 0$, where $L_{i}^{(t)}=-\inf _{0 \leq s \leq t}\left[Z_{i}^{(0)}+\right.$ $\left.X_{i}^{(s)}\right]^{-}$, i.e. $Z_{i}^{(t)}$ is the reflected Lévy process. In terms of dams or queues, $Z_{i}^{(t)}$ constitutes the content or workload process. Throughout, we assume that the system is stable. For the models discussed below, this means that $\phi_{2}^{\prime}(0)>0$.

Model I. (Dams and $M / G / 1$ queues.) The study of queueing models with continuously adaptable service speed $r\left(Z^{(t)}\right)$ goes back to the M/G/1 dam. In such a dam, the output equals $r_{1}$ when the content of the dam is in $(0, K)$ and equals $r_{2}$ when the content of the dam exceeds level $K$. In terms of queueing models, this corresponds to a queue with workload-dependent service speed. We consider an extension of the classical M/G/1 dam. In addition to the service speed (output rate), the arrival rate and service requirement distribution may also depend on the amount of work present. In terms of Lévy processes, this corresponds to a reflected process where the Lévy exponent depends on the amount of work present. In particular, the Lévy exponent equals $\phi_{1}(\cdot)$ when the workload is smaller than $K$ (with reflection in 0 ) and it is $\phi_{2}(\cdot)$ otherwise. For the general M/G/1 queue, the Lévy process is a compound Poisson process with an additional negative drift. For this special case, we denote the arrival rate by $\lambda_{i}$, the mean and LST of the service requirement by $\beta_{i}$ and $\beta_{i}(\cdot)$, respectively, and the service speed by $r_{i}, i=1,2$. Hence, the Lévy exponent reads $\phi_{i}(\omega)=r_{i} \omega-\lambda_{i}+\lambda_{i} \beta_{i}(\omega)$.

Model II. (Poisson observer.) Again, we consider a reflected Lévy process without negative jumps and with two different Lévy exponents. An observer sees the amount of work present at Poisson instants, occurring at rate $\xi$, independent of the processes $\left\{X_{i}^{(t)}, t \geq 0\right\}, i=1,2$. The observer regulates the workload process in the following way. When the workload at the observer instant is larger than some fixed value $K>0$, then the process with Lévy exponent 
$\phi_{2}(\cdot)$ is chosen until the subsequent observer instant. If the observer sees a workload smaller than or equal to $K$ then the process with Lévy exponent $\phi_{1}(\cdot)$ is taken.

More precisely, define $t_{0}:=0$, and let $t_{n}, n=1,2, \ldots$, be the $n$th observer epoch. Let $X_{i}(n):=\left\{X_{i}^{(t)}(n), t \geq 0\right\}, i=1,2$ and $n \geq 0$, be the Lévy input process with exponent $\phi_{i}(\cdot)$ during the interval between the $n$th and $(n+1)$ th observations. For fixed $i=1,2$, we assume that $X_{i}(n), n \geq 0$, are independent and identically distributed Lévy processes without negative jumps. The workload process $\left\{Z^{(t)}, t \geq 0\right\}$ is now defined recursively by $Z^{(0)}=0$ and

$$
Z^{(t)}= \begin{cases}Z^{\left(t_{n}\right)}+X_{1}^{\left(t-t_{n}\right)}(n)+L_{1}^{\left(t-t_{n}\right)}(n) & \text { if } t \in\left(t_{n}, t_{n+1}\right] \text { and } Z^{\left(t_{n}\right)} \leq K, \\ Z^{\left(t_{n}\right)}+X_{2}^{\left(t-t_{n}\right)}(n)+L_{2}^{\left(t-t_{n}\right)}(n) & \text { if } t \in\left(t_{n}, t_{n+1}\right] \text { and } Z^{\left(t_{n}\right)}>K,\end{cases}
$$

where $L_{i}^{(t)}(n)=-\inf _{0 \leq s \leq t}\left[Z^{\left(t_{n}\right)}+X_{i}^{(s)}(n)\right]^{-}$for $i=1,2, n \geq 0$, and $t \in\left(t_{n}, t_{n+1}\right]$. Similarly, we define recursively $L^{(0)}=0$ and

$$
L^{(t)}= \begin{cases}L^{\left(t_{n}\right)}+L_{1}^{\left(t-t_{n}\right)}(n) & \text { if } t \in\left(t_{n}, t_{n+1}\right] \text { and } Z^{\left(t_{n}\right)} \leq K, \\ L^{\left(t_{n}\right)}+L_{2}^{\left(t-t_{n}\right)}(n) & \text { if } t \in\left(t_{n}, t_{n+1}\right] \text { and } Z^{\left(t_{n}\right)}>K .\end{cases}
$$

We also consider the process embedded at observer instants. Let $V_{n}$ denote the workload at time $t_{n}, n \geq 0$. Using the PASTA (Poisson arrivals see time averages) property, it follows that the steady-state distribution of $V_{n}$ equals the steady-state distribution of the workload at an arbitrary instant. Let $Z$ denote this steady-state random variable. To describe the one-step transition probabilities of $V_{n}$, we need to determine the distribution and LST of the workload of a Lévy process with exponent $\phi_{i}(\cdot), i=1,2$, after an exponential time (see Theorem 2.2, below).

Let $T$ denote a generic (exponential) interobservation time. Then

$$
V_{n+1}= \begin{cases}Z_{1}^{(T)} \mid Z_{1}^{(0)}=V_{n} & \text { for } 0 \leq V_{n} \leq K \\ Z_{2}^{(T)} \mid Z_{2}^{(0)}=V_{n} & \text { for } V_{n}>K\end{cases}
$$

\subsection{Preliminaries on Lévy processes}

In this subsection we present some results on reflected Lévy processes with Lévy exponent $\phi(\cdot)$ and only positive jumps, excluding the case that the process has monotone paths.

We first introduce the family of so-called scale functions. Scale functions often appear in the study of first-exit times and exit positions; see, e.g. [2], [6]-[8], [24], [27], and [28]. However, we frequently use scale functions to describe the (steady-state) workload behavior. Define, for $s \in \mathbb{R}, \eta(s):=\sup \{\omega \geq 0: \phi(\omega)=s\}$ as the largest root of the equation $\phi(\omega)=s$.

Definition 2.1. For $q \geq 0$, the $q$-scale function $W^{(q)}:(-\infty, \infty) \rightarrow[0, \infty)$ is the unique function whose restriction to $[0, \infty)$ is continuous and has Laplace transform

$$
\int_{0}^{\infty} \mathrm{e}^{-\omega x} W^{(q)}(x) \mathrm{d} x=\frac{1}{\phi(\omega)-q} \quad \text { for } \omega>\eta(q)
$$

and $W^{(q)}(x)=0$ for $x<0$.

We will frequently restrict ourselves to the case in which $q=0$. In this case, $W(\cdot):=$ $W^{(0)}(\cdot)$, which is also often referred to as the scale function. The scale function can be explicitly determined in various special cases. In this paper we restrict ourselves to providing examples 
for the compound Poisson process with negative drift (in which case $W(\cdot)$ can be related to the waiting time distribution in the M/G/1 queue) and Brownian motions. These examples are further discussed in Section 5. We refer to, for example, [20] and [25] for other examples where the scale function is known in explicit form and to [34] for numerical methods. In the remainder, the subscript $i$ is added to the scale function when it is associated with exponent $\phi_{i}(\cdot), i=1,2$, i.e. we write $W_{i}^{(q)}(\cdot)$ and $W_{i}(\cdot)$.

Now, consider the steady-state workload (denoted by $V$ ) of the reflected Lévy process. The formula for its LST is also known as the generalized Pollaczek-Khinchine formula and is presented in the following theorem; see, e.g. [1, Corollary IX.3.4] or [8] and [22].

Theorem 2.1. Consider a Lévy process without negative jumps and with negative drift, i.e. $0<$ $\phi^{\prime}(0)<\infty$. Then, for $\omega \geq 0$,

$$
\mathrm{E}\left[\mathrm{e}^{-\omega V}\right]=\phi^{\prime}(0) \frac{\omega}{\phi(\omega)} .
$$

The distribution of the steady-state amount of work may be expressed in terms of the scale function. Using Definition 2.1 and partial integration, we obtain

$$
\frac{\omega}{\phi(\omega)}=\omega \int_{0}^{\infty} \mathrm{e}^{-\omega x} W(x) \mathrm{d} x=W(0)+\int_{0^{+}}^{\infty} \mathrm{e}^{-\omega x} \mathrm{~d} W(x) .
$$

Hence, the transform in Theorem 2.1 may be readily inverted, providing

$$
\mathrm{P}(V<x)=\phi^{\prime}(0) W(x) .
$$

In fact, a similar result holds in the case where there is reflection at both 0 and some level $K>0$. In the M/G/1 setting this model is often referred to as the finite dam. Note that reflection at $K$ implies that 'customers' are admitted according to partial rejection; customers arriving at the system that cause an overflow over $K$ are only partly accepted such that the workload equals $K$. Denote by $V^{K}$ the steady-state workload. In [28] it was shown that, in the case of a Lévy process without negative jumps, a similar proportionality result holds as for the finite dam, that is, for $x \in(0, K]$,

$$
\mathrm{P}\left(V^{K}<x\right)=\frac{W(x)}{W(K)} .
$$

Finally, in the model with a Poisson observer, we also need to determine the level of the process after an exponential time; see, e.g. (2.1). Let $T \sim \exp (\xi)$. Starting at $v$ at time 0 , the LST of the workload after $T$ can be found in, for example, [8, Theorem 4b] or [10, Theorem 2].

Theorem 2.2. For $\omega \geq 0$, we have

$$
\mathrm{E}\left[\exp \left\{-\omega Z_{i}^{(T)}\right\} \mid Z_{i}^{(0)}=v\right]=\frac{\xi}{\xi-\phi_{i}(\omega)}\left(\mathrm{e}^{-\omega v}-\omega \frac{\exp \left\{-\eta_{i}(\xi) v\right\}}{\eta_{i}(\xi)}\right),
$$

where $\eta_{i}(\xi)$ is the unique positive zero of $\xi-\phi_{i}(\omega)$.

Remark 2.1. The result of [8] is in fact in terms of the LST of the transient behavior of a reflected process, which is however directly related to the LST of the workload after an exponential time. This follows from the observation that $E\left[\exp \left\{-\omega Z_{i}^{(T)}\right\}\right]$ corresponds to $\xi$ times the double transform of the transient workload behavior, where we take $\xi$ for the parameter of the transform with respect to time. Thus, in the special case of the M/G/1 queue, the above result can also be obtained from, for example, [29, Equation (2.62)]. Similar to [29, Section 2.3], we also have $\mathrm{P}\left(Z_{i}^{(T)}=0 \mid Z_{i}^{(0)}=v\right)=\xi \exp \left\{-\eta_{i}(\xi) v\right\} /\left(\eta_{i}(\xi) r_{i}\right)$. 
A useful relation between the steady-state workload during an exponential interval and the amount of work after an exponential time follows from PASTA; see also [10, Equation (3.4)]. In particular, for $i=1,2$,

$$
\mathrm{E}\left[\exp \left\{-\omega Z_{i}^{(T)}\right\} \mid Z_{i}^{(0)}=v\right]=\frac{1}{\mathrm{E}[T]} \mathrm{E}\left[\int_{s=0}^{T} \exp \left\{-\omega Z_{i}^{(s)}\right\} \mathrm{d} s \mid Z_{i}^{(0)}=v\right] .
$$

The distribution of the workload after an exponential time starting from $v \geq 0$ can also be expressed in terms of scale functions. Let $W_{i}^{(T)}(x ; v)=\mathrm{P}\left(Z_{i}^{(T)} \leq x \mid Z_{i}^{(0)}=v\right)$ and define $\bar{W}_{i}^{(q)}(x):=\int_{0}^{x} W_{i}^{(q)}(y) \mathrm{d} y$, for $i=1$, 2. Applying Laplace inversion, we obtain from Theorem 2.2, for $x \geq 0$,

$$
W_{i}^{(T)}(x ; v)=W_{i}^{(\xi)}(x) \xi \frac{\exp \left\{-\eta_{i}(\xi) v\right\}}{\eta_{i}(\xi)}-\xi \bar{W}_{i}^{(\xi)}(x-v) \mathbf{1}(x \geq v),
$$

where $\mathbf{1}(\cdot)$ denotes the indicator function.

\section{Solution procedure}

In this section we outline the solution procedure that can be generally used to determine the steady-state behavior of systems with two service rates or Lévy exponents. We apply this procedure to various models in Sections 4-6.

Let $Z$ denote the random variable of interest (in our examples, the workload), with LST

$$
\zeta(\omega):=\int_{0}^{\infty} \mathrm{e}^{-\omega x} \mathrm{dP}(Z<x)
$$

The procedure for determining the distribution of $Z$ builds upon techniques applied in [13], $[14$, p. 556], and [17], where the workload in an M/G/1 queue with continuously adaptable service speed is studied. The formal outline presented below is based on [4].

Step 0. Determine two sets of equations for $\zeta(\omega)$; one involving $\zeta(\omega)$ and the incomplete LST $\int_{K}^{\infty} \mathrm{e}^{-\omega x} \mathrm{dP}(Z<x)$, and the other involving $\zeta(\omega)$ and the incomplete LST $\int_{0}^{K} \mathrm{e}^{-\omega x} \mathrm{dP}(Z<x)$ :

$$
\begin{aligned}
& \zeta(\omega)=F_{1}(\omega)+G_{1}(\omega) \int_{K}^{\infty} \mathrm{e}^{-\omega x} \mathrm{dP}(Z<x), \\
& \zeta(\omega)=F_{2}(\omega)+G_{2}(\omega) \int_{0}^{K} \mathrm{e}^{-\omega x} \mathrm{dP}(Z<x),
\end{aligned}
$$

for functions $F_{i}(\cdot)$ and $G_{i}(\cdot), i=1,2$. The precise forms depend on the specific model.

Step 1. Rewrite (3.2) such that $G_{1}(\omega) \int_{K}^{\infty} \mathrm{e}^{-\omega x} \mathrm{dP}(Z<x)$ is the sum of an LST with mass only on $[K, \infty)$ and an LST that depends only on $\mathrm{P}(Z<x)$ for $x>K$ through a constant.

Step 2. Apply Laplace inversion to the reformulated (3.2) resulting from step 1, to determine $\mathrm{P}(Z<x)$ for $x \in(0, K]$.

Step 3. Calculate $\int_{0}^{K} \mathrm{e}^{-\omega x} \mathrm{dP}(Z<x)$ using step 2 and substitute into (3.3), yielding $\zeta(\omega)$. Applying Laplace inversion again, we determine $\mathrm{P}(Z<x)$ for $x>K$.

Step 4. Find the remaining constants by normalization. 
The easiest application of this solution procedure is to the M/G/1 queue where the service speed is continuously adapted based on the workload. This model was analyzed in, for instance, [13], [14, Section III.5.10], and [17]. We rederive their result in Subsection 4.1 in the context of dams with nondecreasing Lévy input and two output rates to demonstrate the basic features of each step in the solution procedure.

While focusing in this paper on reflecting Lévy processes with only positive jumps, we believe that a similar procedure can also be applied to derive steady-state results and first-exit probabilities for Lévy processes with two exponents and only negative jumps. This may be relevant for financial models; see, e.g. [1, Chapter XIV] or [2], [18], [19], [23], [26], [30], and the references therein. The solution procedure then has to be slightly adapted. Intuitively, it should be clear that the process on the interval $(K, \infty)$ is affected only by the behavior of the process on $(0, K]$ through a constant. Hence, in step 2 we should first apply Laplace inversion on $(K, \infty)$ and then use that result in step 3 to determine the behavior on $(0, K]$, i.e. steps 2 and 3 are reversed. Accordingly, in step $1, G_{2}(\omega) \int_{0}^{K} \mathrm{e}^{-\omega x} \mathrm{dP}(Z<x)$ should be rewritten as the sum of an LST with mass only on $(0, K]$ and an LST that does depend only on $\mathrm{P}(Z<x)$ for $x \leq K$ through a constant.

\section{Dam processes and $M / G / 1$ queues}

In this section we consider (a generalization of) the classical M/G/1 dam. First, we derive the steady-state workload distribution for the dam with two release rates (service speeds). This concerns an instructive application of the solution procedure, giving insight into the fundamentals of each step. Second, we consider a general M/G/1 queue, where the service speed, arrival rate, and service requirement distribution may depend on the amount of work present.

For dam processes, the input is a nondecreasing Lévy process (a subordinator); see, e.g. [11], [15], [30, Chapter 3], or [32]. The release rate here depends on the content of the dam determined by a single threshold (Model I). Before considering the two separate models, we start by deriving two sets of equations for dams and the general M/G/1 queue.

Step 0: Determining the equations. For dams and M/G/1-type models, we consider downcrossings of level $K$ as regeneration epochs. Let $Z^{(0)}=K$. Define $\tau_{1}:=\inf \left[t>0: Z^{(t)} \geq\right.$ $K]$ as the first upcrossing of level $K$ and $\tau_{2}:=\inf \left[t \geq \tau_{1}: Z^{(t)}=K\right]$ as the subsequent downcrossing. It then follows directly from [32] that $0<\tau_{1}<\tau_{2}$ (almost surely). The content process can thus be considered as a regenerative process, and has a stationary distribution. We interpret this model as a regenerative alternating Lévy process, as described in Appendix A.

Since there is no reflection during the second part of the cycle, $\mathrm{E}\left[L_{2}^{\left(\tau_{2}-\tau_{1}\right)}\right]=0$. Note that $\mathrm{E}\left[\exp \left\{-\omega Z^{\left(\tau_{2}\right)}\right\}\right]=\mathrm{E}\left[\exp \left\{-\omega Z^{(0)}\right\}\right]=\mathrm{e}^{-\omega K}$. According to the theory of regenerative processes,

$$
\begin{aligned}
& \int_{0}^{K} \mathrm{e}^{-\omega x} \mathrm{dP}(Z<x)=\frac{1}{\mathrm{E}\left[\tau_{2}\right]} \mathrm{E}\left[\int_{s=0}^{\tau_{1}} \exp \left\{-\omega Z^{(s)}\right\} \mathrm{d} s\right] \\
& \int_{K}^{\infty} \mathrm{e}^{-\omega x} \mathrm{dP}(Z<x)=\frac{1}{\mathrm{E}\left[\tau_{2}\right]} \mathrm{E}\left[\int_{s=\tau_{1}}^{\tau_{2}} \exp \left\{-\omega Z^{(s)}\right\} \mathrm{d} s\right] .
\end{aligned}
$$

Combining the above yields two equations for $\zeta(\cdot)$ from Lemma A.1 (see Appendix A):

$$
\zeta(\omega)=\frac{\mathrm{E}\left[L_{1}^{\left(\tau_{1}\right)}\right]}{\mathrm{E}\left[\tau_{2}\right]} \frac{\omega}{\phi_{2}(\omega)}+\frac{\phi_{2}(\omega)-\phi_{1}(\omega)}{\phi_{2}(\omega)} \int_{0}^{K} \mathrm{e}^{-\omega x} \mathrm{dP}(Z<x)
$$


and

$$
\zeta(\omega)=\frac{\mathrm{E}\left[L_{1}^{\left(\tau_{1}\right)}\right]}{\mathrm{E}\left[\tau_{2}\right]} \frac{\omega}{\phi_{1}(\omega)}+\frac{\phi_{1}(\omega)-\phi_{2}(\omega)}{\phi_{1}(\omega)} \int_{K}^{\infty} \mathrm{e}^{-\omega x} \mathrm{dP}(Z<x) .
$$

Hence, (4.1) and (4.2) correspond to (3.3) and (3.2), respectively, where

$$
F_{i}(\omega)=\frac{\mathrm{E}\left[L_{1}^{\left(\tau_{1}\right)}\right] \omega}{\phi_{i}(\omega) \mathrm{E}\left[\tau_{2}\right]} \quad \text { and } \quad G_{i}(\omega)=\frac{\phi_{i}(\omega)-\phi_{3-i}(\omega)}{\phi_{i}(\omega)}, \quad i=1,2 .
$$

\subsection{Dams: change of drift}

First assume that $X_{i}(t)=X(t)-r_{i} t, i=1,2$, with $\{X(t), t \geq 0\}$ a subordinator. This concerns the classical dam where only the release rate is adapted, which reduces to an M/G/1 queue with two service speeds in the case where $X(t)$ is a compound Poisson process; see also [13], [14, Section III.5.10], and [17].

Step 1: Rewriting (4.2). Using the fact that $\left(\phi_{1}(\omega)-\phi_{2}(\omega)\right) / \phi_{1}(\omega)=\left(r_{1}-r_{2}\right) \omega / \phi_{1}(\omega)$,

$$
\zeta(\omega)=\frac{\mathrm{E}\left[L_{1}^{\left(\tau_{1}\right)}\right]}{\mathrm{E}\left[\tau_{2}\right]} \frac{\omega}{\phi_{1}(\omega)}+\left(r_{1}-r_{2}\right) \frac{\omega}{\phi_{1}(\omega)} \int_{K}^{\infty} \mathrm{e}^{-\omega x} \mathrm{dP}(Z<x) .
$$

Step 2: Workload distribution on $(0, K]$. The first term on the right-hand side of (4.3) can be readily inverted using Theorem 2.1 and Definition 2.1, yielding the scale function $W_{1}(\cdot)$. We refer the reader to [15] for details on the exact form of $W_{1}(\cdot)$; see also Subsection 5.2 for the $\mathrm{M} / \mathrm{G} / 1$ case. The second term involves a convolution, corresponding to the sum of two functions. Since the Laplace inverse of $\omega / \phi_{1}(\omega)$ has mass on $[0, \infty)$ and $\int_{K}^{\infty} \mathrm{e}^{-\omega x} \mathrm{dP}(Z<x)$ is (up to a constant) the LST of a function with mass on $[K, \infty)$, its convolution has mass only on $[K, \infty)$. Hence, for $x \in(0, K]$, the Laplace inverse of $\zeta(\omega)$ is given by

$$
\mathrm{P}(Z<x)=\frac{\mathrm{E}\left[L_{1}^{\left(\tau_{1}\right)}\right]}{\mathrm{E}\left[\tau_{2}\right]} W_{1}(x) .
$$

Step 3: Workload distribution on $(K, \infty)$. Apply Laplace inversion to (4.1) to obtain the distribution of $Z$ on $(K, \infty)$. The inverse of the first transform on the right-hand side of (4.1) is given by the scale function $W_{2}(\cdot)$. Note that $\left(\phi_{2}(\omega)-\phi_{1}(\omega)\right) / \phi_{2}(\omega)$ equals $\left(r_{2}-r_{1}\right) \omega / \phi_{2}(\omega)$, which is $\left(r_{2}-r_{1}\right)$ times the LST of the scale function $W_{2}(\cdot)$. The second term now involves an incomplete convolution. Using the result for the distribution of $Z$ on $(0, K]$, i.e. (4.4), we obtain, for $x>K$,

$$
\mathrm{P}(Z<x)=\frac{\mathrm{E}\left[L_{1}^{\left(\tau_{1}\right)}\right]}{\mathrm{E}\left[\tau_{2}\right]}\left(W_{2}(x)+\left(r_{2}-r_{1}\right) \int_{0}^{K} W_{2}(x-y) \mathrm{d} W_{1}(y)\right) .
$$

Step 4: Determination of the constant. Use normalization and (4.4). More specifically, letting $\omega \downarrow 0$ in (4.1) and applying (4.4) with $x=K$, we obtain

$$
\frac{\mathrm{E}\left[L_{1}^{\left(\tau_{1}\right)}\right]}{\mathrm{E}\left[\tau_{2}\right]}=\frac{\phi_{2}^{\prime}(0)}{1+\left(r_{2}-r_{1}\right) W_{1}(K)} .
$$

Using the specific form of $W_{i}(\cdot)$ given in Subsection 5.2, it may be readily checked that the results coincide with the results in [13], [14, p. 556], and [17]. 
Remark 4.1. Recently, a similar model has been studied in the context of insurance risk processes [23]. The above expressions for the workload distribution coincide with the expression for the survival probability in the dual insurance risk model (there called refracted Lévy processes, i.e. Lévy processes whose dynamics change by subtracting a fixed linear drift whenever the process is above a prespecified level); see [23, Theorem 5].

\subsection{General $M / G / 1$ queue}

We now apply the procedure to the workload in the general M/G/1 queue with arrival rates $\lambda_{i}$, service requirement LSTs $\beta_{i}(\cdot)$, and service speeds $r_{i}, i=1,2$. The Lévy exponent then reduces to $\phi_{i}(\omega)=r_{i} \omega-\lambda_{i}+\lambda_{i} \beta_{i}(\omega)$ for $i=1,2$. Define $\rho_{i}:=\lambda_{i} \beta_{i} / r_{i}$. The stability condition for this case reads $\rho_{2}<1$.

Step 1: Rewriting (4.2). Using the fact that $\phi_{i}(\omega)=r_{i} \omega-\lambda_{i}+\lambda_{i} \beta_{i}(\omega), i=1,2$, we may rewrite the fraction of Lévy exponents as follows:

$$
\begin{aligned}
\frac{\phi_{1}(\omega)-\phi_{2}(\omega)}{\phi_{1}(\omega)}= & \frac{\left(r_{1}-r_{2}\right) \omega+\left(\lambda_{2} / \lambda_{1}\right)\left(-\lambda_{1} \beta_{2}(\omega)+\lambda_{1} \beta_{1}(\omega)\right)}{\phi_{1}(\omega)} \\
& +\left(1-\frac{\lambda_{2}}{\lambda_{1}}\right) \frac{-\lambda_{1}+\lambda_{1} \beta_{1}(\omega)}{\phi_{1}(\omega)} \\
= & -r_{2} \frac{\omega}{\phi_{1}(\omega)}+\frac{\lambda_{2}}{\lambda_{1}} \frac{r_{1} \omega-\lambda_{1} \beta_{2}(\omega)+\lambda_{1} \beta_{1}(\omega)}{r_{1} \omega-\lambda_{1}+\lambda_{1} \beta_{1}(\omega)}+1-\frac{\lambda_{2}}{\lambda_{1}} .
\end{aligned}
$$

Although this form is rather involved, we have decomposed it into three familiar terms. The first one is related to the standard M/G/1 queue, the second one corresponds to an M/G/1 queue with exceptional first service (up to a constant) (see, e.g. [35], [36], or Example A.1), and the third is just a constant. Substituting the above into (4.2) provides

$$
\begin{aligned}
\zeta(\omega)=\frac{\mathrm{E}\left[L_{1}^{\left(\tau_{1}\right)}\right]}{\mathrm{E}\left[\tau_{2}\right]} \frac{\omega}{\phi_{1}(\omega)}+( & -r_{2} \frac{\omega}{\phi_{1}(\omega)}+\frac{\lambda_{2}}{\lambda_{1}} \frac{r_{1} \omega-\lambda_{1} \beta_{2}(\omega)+\lambda_{1} \beta_{1}(\omega)}{r_{1} \omega-\lambda_{1}+\lambda_{1} \beta_{1}(\omega)} \\
& \left.+1-\frac{\lambda_{2}}{\lambda_{1}}\right) \int_{K}^{\infty} \mathrm{e}^{-\omega x} \mathrm{dP}(Z<x)
\end{aligned}
$$

Step 2: Workload distribution on $(0, K]$. The first term on the right-hand side of (4.5) can be readily inverted using Theorem 2.1 and Definition 2.1, yielding the scale function $W_{1}(\cdot)$; see also Subsection 5.2. For the remaining terms, we apply the decomposition of step 1 and consider each term separately. The first decomposed term, $\left(\omega / \phi_{1}(\omega)\right) \int_{K}^{\infty} \mathrm{e}^{-\omega x} \mathrm{dP}(Z<x)$, corresponds to the convolution of two functions. Because the inverse of $\omega / \phi_{1}(\omega)$ has mass on $[0, \infty)$ and $\int_{K}^{\infty} \mathrm{e}^{-\omega x} \mathrm{dP}(Z<x)$ is the transform of a function with mass on $[K, \infty)$, this convolution has mass on $[K, \infty)$.

The term $\left(r_{1} \omega-\lambda_{1} \beta_{2}(\omega)+\lambda_{1} \beta_{1}(\omega)\right) /\left(r_{1} \omega-\lambda_{1}+\lambda_{1} \beta_{1}(\omega)\right)$ is the Laplace transform of the workload in a queue with exceptional first service requirement (see, e.g. [35], [36], or Example A.1) and, thus, the inverse has mass on $[0, \infty)$. The convolution with $\mathrm{P}(Z<x)$ on $[K, \infty)$ clearly has no mass on $[0, K)$. For the final term, it is readily seen that it has mass only on $[K, \infty)$. Hence, for $x \in(0, K]$,

$$
\mathrm{P}(Z<x)=\frac{\mathrm{E}\left[L_{1}^{\left(\tau_{1}\right)}\right]}{\mathrm{E}\left[\tau_{2}\right]} W_{1}(x),
$$

which is proportional to regular M/G/1 behavior with $\phi_{1}(\omega)=r_{1} \omega-\lambda_{1}+\lambda_{1} \beta_{1}(\omega)$. 
Step 3: Workload distribution on $(K, \infty)$. We apply (4.1) and a similar decomposition as in step 1 to obtain the workload distribution on $(K, \infty)$. In particular, (4.1) may be equivalently written as

$$
\begin{aligned}
\zeta(\omega)= & \frac{\mathrm{E}\left[L_{1}^{\left(\tau_{1}\right)}\right]}{\mathrm{E}\left[\tau_{2}\right]} \frac{\omega}{\phi_{2}(\omega)} \\
& +\left(-r_{1} \frac{\omega}{\phi_{2}(\omega)}+\frac{\lambda_{1}}{\lambda_{2}} \frac{r_{2} \omega-\lambda_{2} \beta_{1}(\omega)+\lambda_{2} \beta_{2}(\omega)}{r_{2} \omega-\lambda_{2}+\lambda_{2} \beta_{2}(\omega)}+1-\frac{\lambda_{1}}{\lambda_{2}}\right) \int_{0}^{K} \mathrm{e}^{-\omega x} \mathrm{dP}(Z<x) .
\end{aligned}
$$

Since we have determined $\mathrm{P}(Z<x)$ for $x \in(0, K]$, we next apply Laplace inversion to each of the above terms separately. Note that the inverse of the first term is given by the scale function $W_{2}(\cdot)$ (again, see Subsection 5.2 for the precise form of $W_{2}(x)$ ). The second term involves the convolution of $W_{2}(\cdot)$ and the distribution of $Z$ on $(0, K]$, giving $\int_{0}^{K} W_{2}(x-y) \mathrm{d} W_{1}(y)$ times a constant. For the third transform, we introduce $W_{2}^{\text {exc }}(\cdot)$ as the distribution of the workload in an M/G/1 queue with service rate $r_{2}$, arrival rate $\lambda_{2}$, and generic service requirement $B_{2}$, but with exceptional first service $B_{1}$ in a busy period. Similar to the second term we obtain the convolution $\int_{0}^{K} W_{2}^{\text {exc }}(x-y) \mathrm{d} W_{1}(y)$ up to a constant. (For the constant, multiply and divide by $\left(1-\rho_{2}\right) /\left(1+\lambda_{2} \beta_{1} / r_{2}-\rho_{2}\right)$.) The final term corresponds to the transform of a function with mass only on $(0, K]$. Hence, for $x>K$, the inverse appears only as the constant $\mathrm{P}(Z<K)$.

Summarizing the above we have, for $x>K$,

$$
\begin{aligned}
\mathrm{P}(Z<x)=\frac{\mathrm{E}\left[L_{1}^{\left(\tau_{1}\right)}\right]}{\mathrm{E}\left[\tau_{2}\right]}( & W_{2}(x)-r_{1} \int_{0}^{K} W_{2}(x-y) \mathrm{d} W_{1}(y) \\
& +\frac{\lambda_{1}}{\lambda_{2}} \frac{1+\lambda_{2} \beta_{1} / r_{2}-\rho_{2}}{1-\rho_{2}} \int_{0}^{K} W_{2}^{\operatorname{exc}}(x-y) \mathrm{d} W_{1}(y) \\
& \left.+\left(1-\frac{\lambda_{1}}{\lambda_{2}}\right) W_{1}(K)\right)
\end{aligned}
$$

Step 4: Determination of the constant. Use normalization and (4.6). In particular, letting $\omega \downarrow 0$ in (4.1) and using (4.6) with $x=K$, we obtain

$$
\frac{\mathrm{E}\left[L_{1}^{\left(\tau_{1}\right)}\right]}{\mathrm{E}\left[\tau_{2}\right]}=\frac{r_{2}\left(1-\rho_{2}\right)}{1+\left(r_{2}\left(1-\rho_{2}\right)-r_{1}\left(1-\rho_{1}\right)\right) W_{1}(K)},
$$

where the specific form of $W_{1}(\cdot)$ is given in Subsection 5.2.

\section{Lévy processes with Poisson observer}

In this section we consider the reflected Lévy process where the Lévy exponent is adapted at Poisson instants; see Section 2. To determine the steady-state workload distribution, we use steps $0-4$ outlined in Section 3. In particular, steps 0 and 1 are obtained using a direct approach. Depending on the form of $\left(\phi_{i}(\omega)-\phi_{3-i}(\omega)\right) / \phi_{i}(\omega), i=1,2$, it is possible to give a direct and intuitive derivation of steps $2-4$, leading to intuitively appealing expressions for the steady-state workload distribution in various special cases: in Subsections 5.1, 5.2, and 5.3 we respectively consider the special case of a change of drift, the M/G/1 case (i.e. compound Poisson with a negative drift), and the case of Brownian motion.

In general, it is not possible to rewrite $\left(\phi_{i}(\omega)-\phi_{3-i}(\omega)\right) / \phi_{i}(\omega)$ in tractable terms. The steady-state workload distribution for this general case can be found in Section 6. 
In the following lemma we present the two equations for $\zeta(\cdot)$ as outlined in the procedure of Section 3. First we introduce the constant $Q$ :

$$
Q:=\xi \int_{0}^{K} \frac{\exp \left\{-\eta_{1}(\xi) x\right\}}{\eta_{1}(\xi)} \mathrm{dP}(Z<x)+\xi \int_{K}^{\infty} \frac{\exp \left\{-\eta_{2}(\xi) x\right\}}{\eta_{2}(\xi)} \mathrm{dP}(Z<x)
$$

Lemma 5.1. With $\eta_{i}(\xi), i=1,2$, defined in Theorem 2.2, for $\operatorname{Re}(\omega) \geq 0$,

$$
\begin{aligned}
\zeta(\omega)=Q \frac{\omega}{\phi_{1}(\omega)}+ & \frac{\phi_{1}(\omega)-\phi_{2}(\omega)}{\phi_{1}(\omega)} \frac{\xi}{\xi-\phi_{2}(\omega)} \\
& \times\left(\int_{K}^{\infty} \mathrm{e}^{-\omega x} \mathrm{dP}(Z<x)-\omega \int_{K}^{\infty} \frac{\exp \left\{-\eta_{2}(\xi) x\right\}}{\eta_{2}(\xi)} \mathrm{dP}(Z<x)\right)
\end{aligned}
$$

and

$$
\begin{aligned}
\zeta(\omega)=Q \frac{\omega}{\phi_{2}(\omega)}+ & \frac{\phi_{2}(\omega)-\phi_{1}(\omega)}{\phi_{2}(\omega)} \frac{\xi}{\xi-\phi_{1}(\omega)} \\
& \times\left(\int_{0}^{K} \mathrm{e}^{-\omega x} \mathrm{dP}(Z<x)-\omega \int_{0}^{K} \frac{\exp \left\{-\eta_{1}(\xi) x\right\}}{\eta_{1}(\xi)} \mathrm{dP}(Z<x)\right) .
\end{aligned}
$$

Proof. Our starting point is the recursion relation (2.1) between $V_{n}$ and $V_{n+1}$, where $V_{n}$ denotes the workload just before the $n$th observer instant. Condition on $V_{n}$, use (2.1), and apply Theorem 2.2, to obtain

$$
\begin{aligned}
\mathrm{E}\left[\exp \left\{-\omega V_{n+1}\right\}\right] & \\
= & \int_{0}^{\infty} \mathrm{E}\left[\exp \left\{-\omega V_{n+1}\right\} \mid V_{n}=x\right] \mathrm{dP}\left(V_{n}<x\right) \\
= & \frac{\xi}{\xi-\phi_{1}(\omega)}\left(\int_{0}^{K} \mathrm{e}^{-\omega x} \mathrm{dP}\left(V_{n}<x\right)-\omega \int_{0}^{K} \frac{\exp \left\{-\eta_{1}(\xi) x\right\}}{\eta_{1}(\xi)} \mathrm{dP}\left(V_{n}<x\right)\right) \\
& +\frac{\xi}{\xi-\phi_{2}(\omega)}\left(\int_{K}^{\infty} \mathrm{e}^{-\omega x} \mathrm{dP}\left(V_{n}<x\right)-\omega \int_{K}^{\infty} \frac{\exp \left\{-\eta_{2}(\xi) x\right\}}{\eta_{2}(\xi)} \mathrm{dP}\left(V_{n}<x\right)\right) .
\end{aligned}
$$

To analyze the steady-state behavior of $V_{n}$, we let $n \rightarrow \infty$. Using the PASTA property, it follows that the steady-state distribution of $V_{n}$ equals the distribution of the workload at an arbitrary instant $\mathrm{P}(Z<x)$. Using the above, we may obtain two alternative equations for $\zeta(\omega)$, as described in step 0 of the general procedure: one involving the incomplete LST $\int_{K}^{\infty} \mathrm{e}^{-\omega x} \mathrm{dP}(Z<x)$ and one involving the incomplete $\operatorname{LST} \int_{0}^{K} \mathrm{e}^{-\omega x} \mathrm{dP}(Z<x)$.

Note that, for $i=1,2$,

$$
1-\frac{\xi}{\xi-\phi_{i}(\omega)}=\frac{-\phi_{i}(\omega)}{\xi-\phi_{i}(\omega)}
$$

Then, for the first equation, we deduce, after some basic manipulations, using (3.1) and (5.4) 
for $i=1$, and dividing by the term on the right-hand side of (5.4) with $i=1$, that

$$
\begin{aligned}
\zeta(\omega)= & \frac{\omega}{\phi_{1}(\omega)} \xi \int_{0}^{K} \frac{\exp \left\{-\eta_{1}(\xi) x\right\}}{\eta_{1}(\xi)} \mathrm{dP}(Z<x) \\
& +\frac{\omega}{\phi_{1}(\omega)} \frac{\xi-\phi_{1}(\omega)}{\xi-\phi_{2}(\omega)} \xi \int_{K}^{\infty} \frac{\exp \left\{-\eta_{2}(\xi) x\right\}}{\eta_{2}(\xi)} \mathrm{dP}(Z<x) \\
& +\frac{1}{\phi_{1}(\omega)}\left(1-\frac{\xi-\phi_{1}(\omega)}{\xi-\phi_{2}(\omega)}\right) \xi \int_{K}^{\infty} \mathrm{e}^{-\omega x} \mathrm{dP}(Z<x) \\
= & \frac{\omega}{\phi_{1}(\omega)}\left(\xi \int_{0}^{K} \frac{\exp \left\{-\eta_{1}(\xi) x\right\}}{\eta_{1}(\xi)} \mathrm{dP}(Z<x)+\xi \int_{K}^{\infty} \frac{\exp \left\{-\eta_{2}(\xi) x\right\}}{\eta_{2}(\xi)} \mathrm{dP}(Z<x)\right) \\
& +\frac{\phi_{1}(\omega)-\phi_{2}(\omega)}{\phi_{1}(\omega)} \frac{\xi-\phi_{2}(\omega)}{\xi}\left(\int_{K}^{\infty} \mathrm{e}^{-\omega x} \mathrm{dP}(Z<x)\right. \\
& \left.\quad-\omega \int_{K}^{\infty} \frac{\exp \left\{-\eta_{2}(\xi) x\right\}}{\eta_{2}(\xi)} \mathrm{dP}(Z<x)\right) .
\end{aligned}
$$

Using (3.1) and (5.4) for $i=2$, and dividing by the term on the right-hand side of (5.4) with $i=2$, we similarly obtain, after some calculations,

$$
\begin{aligned}
\zeta(\omega)= & \frac{\omega}{\phi_{2}(\omega)} \frac{\xi-\phi_{2}(\omega)}{\xi-\phi_{1}(\omega)} \xi \int_{0}^{K} \frac{\exp \left\{-\eta_{1}(\xi) x\right\}}{\eta_{1}(\xi)} \mathrm{dP}(Z<x) \\
& +\frac{\omega}{\phi_{2}(\omega)} \xi \int_{K}^{\infty} \frac{\exp \left\{-\eta_{2}(\xi) x\right\}}{\eta_{2}(\xi)} \mathrm{dP}(Z<x) \\
& +\frac{1}{\phi_{2}(\omega)}\left(1-\frac{\xi-\phi_{2}(\omega)}{\xi-\phi_{1}(\omega)}\right) \xi \int_{0}^{K} \mathrm{e}^{-\omega x} \mathrm{dP}(Z<x) \\
= & \frac{\omega}{\phi_{2}(\omega)}\left(\xi \int_{0}^{K} \frac{\exp \left\{-\eta_{1}(\xi) x\right\}}{\eta_{1}(\xi)} \mathrm{dP}(Z<x)+\xi \int_{K}^{\infty} \frac{\exp \left\{-\eta_{2}(\xi) x\right\}}{\eta_{2}(\xi)} \mathrm{dP}(Z<x)\right) \\
& +\frac{\phi_{2}(\omega)-\phi_{1}(\omega)}{\phi_{2}(\omega)} \frac{\xi}{\xi-\phi_{1}(\omega)}\left(\int_{0}^{K} \mathrm{e}^{-\omega x} \mathrm{dP}(Z<x)\right. \\
& \left.-\omega \int_{0}^{K} \frac{\exp \left\{-\eta_{1}(\xi) x\right\}}{\eta_{1}(\xi)} \mathrm{dP}(Z<x)\right) .
\end{aligned}
$$

Combining (5.1) with (5.5) and (5.6) yields (5.2) and (5.3), respectively. This completes the proof.

Step 1: Rewriting (5.2). Rewrite the second term on the right-hand side of (5.2) into the sum of two LSTs: an LST with mass only on $[K, \infty)$ and an LST that depends only on the distribution of $Z$ through a constant. This step is based on the following intuition. Consider the workload process continuous in time and note that a Lévy exponent $\phi_{2}(\cdot)$ implies that the workload at the previous observer instant was larger than $K$. When the observer sees a workload smaller than $K$, then the exponent is set to $\phi_{1}(\cdot)$. Thus, periods with exponent $\phi_{2}(\cdot)$ and workloads smaller than $K$ are always initiated with an observer finding a workload larger than $K$ followed by a downcrossing of level $K$ before the next observer instant. Owing to the lack-of-memory property of the Poisson process, the remaining interobservation time is still exponential at a downcrossing of $K$. Hence, the precise distribution of $Z$ on $(K, \infty)$ affects only the distribution of $Z$ on $[0, K]$ through a constant. 
Applying the above intuition, we condition on $V_{n}>K$ and use Theorem 2.2 to obtain

$$
\begin{aligned}
\int_{K}^{\infty} \mathrm{E}\left[\exp \left\{-\omega V_{n+1}\right\} \mid V_{n}=x\right] \mathrm{dP}\left(V_{n}<x\right) \\
\quad=\frac{\xi}{\xi-\phi_{2}(\omega)}\left(\int_{K}^{\infty} \mathrm{e}^{-\omega x} \mathrm{dP}\left(V_{n}<x\right)-\omega \int_{K}^{\infty} \frac{\exp \left\{-\eta_{2}(\xi) x\right\}}{\eta_{2}(\xi)} \mathrm{dP}\left(V_{n}<x\right)\right) .
\end{aligned}
$$

Observe that the right-hand side corresponds to the final part of the second term on the righthand side of (5.2) in the case $n \rightarrow \infty$. Let $\tau_{K}:=\inf \left[t \geq 0: Z^{(t)}=K\right]$ be the first hitting time of $K$. Recall that $T \sim \exp (\xi)$. Using (2.1) in the first step and the lack-of-memory property of Lévy processes and of the observer arrival process in the second step, we deduce that

$$
\begin{aligned}
\int_{K}^{\infty} \mathrm{E} & {\left[\exp \left\{-\omega V_{n+1}\right\} \mid V_{n}=x\right] \mathrm{dP}\left(V_{n}<x\right) } \\
= & \int_{K}^{\infty} \mathrm{E}\left[\exp \left\{-\omega Z_{2}^{(T)}\right\} \mathbf{1}\left(T<\tau_{K}\right) \mid Z_{2}^{(0)}=x\right] \mathrm{dP}\left(V_{n}<x\right) \\
& +\int_{K}^{\infty} \mathrm{E}\left[\exp \left\{-\omega Z_{2}^{(T)}\right\} \mathbf{1}\left(T \geq \tau_{K}\right) \mid Z_{2}^{(0)}=x\right] \mathrm{dP}\left(V_{n}<x\right) \\
= & \int_{K}^{\infty} \mathrm{E}\left[\exp \left\{-\omega Z_{2}^{(T)}\right\} \mathbf{1}\left(T<\tau_{K}\right) \mid Z_{2}^{(0)}=x\right] \mathrm{dP}(Z<x) \\
& +P_{\downarrow K} \mathrm{E}\left[\exp \left\{-\omega Z_{2}^{(T)}\right\} \mid Z_{2}^{(0)}=K\right],
\end{aligned}
$$

where in the final step we let $n \rightarrow \infty$ and use the PASTA property. Here $P_{\downarrow K}$ is the probability of downcrossing level $K$ before an exponential time starting from $x>K$ according to the distribution $\mathrm{P}(Z<x)$. Using results concerning the LST of first-exit times (see, e.g. [24]), it may be checked that

$$
P_{\downarrow K}=\xi \exp \left\{\eta_{2}(\xi) K\right\} \int_{K}^{\infty} \exp \left\{-\eta_{2}(\xi) x\right\} \mathrm{dP}(Z<x) .
$$

Observe that the first term on the right-hand side of (5.7) corresponds to an LST with mass on $[K, \infty)$ and the second term of (5.7) depends only on the distribution of $Z$ on $(K, \infty)$ through a constant. Combining the above, (5.2) can be written as

$$
\begin{aligned}
\zeta(\omega)= & Q \frac{\omega}{\phi_{1}(\omega)}+\frac{\phi_{1}(\omega)-\phi_{2}(\omega)}{\phi_{1}(\omega)} \int_{K}^{\infty} \mathrm{E}\left[\exp \left\{-\omega Z_{2}^{(T)}\right\} \mathbf{1}\left(T<\tau_{K}\right) \mid Z_{2}^{(0)}=x\right] \operatorname{dP}(Z<x) \\
& +\frac{\phi_{1}(\omega)-\phi_{2}(\omega)}{\phi_{1}(\omega)} P_{\downarrow K} \mathrm{E}\left[\exp \left\{-\omega Z_{2}^{(T)}\right\} \mid Z_{2}^{(0)}=K\right],
\end{aligned}
$$

where $\mathrm{E}\left[\exp \left\{-\omega Z_{2}^{(T)}\right\} \mid Z_{2}^{(0)}=K\right]$ may be obtained from Theorem 2.2. This completes step 1 .

We note that $\left(\phi_{i}(\omega)-\phi_{3-i}(\omega)\right) / \phi_{i}(\omega), i=1,2$, cannot be reduced in general. This is the reason for applying alternating Lévy processes as defined in Appendix A for the general case; see Section 6. However, in several important special cases, as in Subsections 5.1-5.3 below, the derivation is more insightful, leading to intuitively appealing expressions.

\subsection{Poisson observer: change of drift}

In this subsection we consider the important special case of a change of drift, i.e. we assume that $\phi_{i}(\omega)=r_{i} \omega+\tilde{\phi}(\omega), i=1,2$, for some Lévy exponent $\tilde{\phi}(\omega)$. So,

$$
\frac{\phi_{1}(\omega)-\phi_{2}(\omega)}{\phi_{1}(\omega)}=\left(r_{1}-r_{2}\right) \frac{\omega}{\phi_{1}(\omega)},
$$

where the right-hand side can be readily inverted using the scale function $W_{1}(\cdot)$. 
Step 2: Workload distribution on $(0, K]$. Apply Laplace inversion to each of the three terms on the right-hand side of (5.8) separately. The inverse of the first term can be directly obtained from Definition 2.1 (see also Theorem 2.1 and (2.2)), yielding $Q W_{1}(\cdot)$.

Using (5.9), the second transform reduces to

$$
\left(r_{1}-r_{2}\right) \frac{\omega}{\phi_{1}(\omega)} \int_{K}^{\infty} \mathrm{E}\left[\exp \left\{-\omega Z_{2}^{(T)}\right\} \mathbf{1}\left(T<\tau_{K}\right) \mid Z_{2}^{(0)}=x\right] \mathrm{dP}(Z<x) .
$$

We note that this involves the product of two LSTs, corresponding to the convolution of two functions. Since the first function has mass on $[0, \infty)$ and the second has mass only on $[K, \infty)$, its convolution has no mass on $[0, K)$.

The third transform on the right-hand side of (5.8) also corresponds to a convolution of two functions. The Laplace inverse of $\left(\omega / \phi_{1}(\omega)\right) \mathrm{E}\left[\exp \left\{-\omega Z_{2}^{(T)}\right\} \mid Z_{2}^{(0)}=K\right] \operatorname{reads} W_{1}(x) *$ $W_{2}^{(T)}(x ; K)$, where '*' denotes a convolution and $W_{2}^{(T)}(x ; K)$ is given by $(2.5)$.

Combining the above, we have, for $x \in(0, K]$,

$$
\mathrm{P}(Z<x)=Q W_{1}(x)+\left(r_{1}-r_{2}\right) P_{\downarrow K} \int_{0}^{x} W_{2}^{(T)}(x-y ; K) \mathrm{d} W_{1}(y) .
$$

Step 3: Workload distribution on $(K, \infty)$. Substituting the results from step 1 into $(5.3)$ provides $\zeta(\omega)$. In this step we use (5.3) directly to derive the workload distribution on $(K, \infty)$.

For the first term on the right-hand side of (5.3), the Laplace inverse is given by the scale function $W_{2}(\cdot)$. For the special case of a change of drift, the second term reduces to

$$
\left(r_{2}-r_{1}\right) \frac{\omega}{\phi_{2}(\omega)} \frac{\xi}{\xi-\phi_{1}(\omega)}\left(\int_{0}^{K} \mathrm{e}^{-\omega x} \mathrm{dP}(Z<x)-\omega \int_{0}^{K} \frac{\exp \left\{-\eta_{1}(\xi) x\right\}}{\eta_{1}(\xi)} \mathrm{dP}(Z<x)\right) .
$$

Inversion of the above term corresponds to a convolution of the scale function $W_{2}(\cdot)$ (given by $\left.\omega / \phi_{2}(\omega)\right)$ and a second function. For the latter one, we note that, by applying Theorem 2.2, the inverse is given by

$$
\tilde{W}^{(T)}(x):=\int_{0}^{K} W_{1}^{(T)}(x ; y) \mathrm{dP}(Z<y),
$$

i.e. the amount of work of a reflected Lévy process with exponent $\phi_{1}(\omega)$ after an exponential time, starting according to $\mathrm{P}(Z<x)$. Since we have determined the distribution of $Z$ on $[0, K]$, we have also found $\tilde{W}^{(T)}(\cdot)$ (although its precise form may be involved).

Combining the above, we obtain, for $x>K$,

$$
\mathrm{P}(Z<x)=Q W_{2}(x)+\left(r_{2}-r_{1}\right) \int_{0}^{x} W_{2}(x-y) \mathrm{d} \tilde{W}^{(T)}(y) .
$$

Step 4: Determination of the constants. The remaining constants can be determined in a similar fashion as in [4]. First, the constants

$$
\int_{0}^{K} \exp \left\{-\eta_{1}(\xi) x\right\} \mathrm{dP}(Z<x) \text { and } \int_{K}^{\infty} \exp \left\{-\eta_{2}(\xi) x\right\} \mathrm{dP}(Z<x)
$$

can be expressed in terms of $Q$ and $\mathrm{P}(Z<K)$ using (5.1) and (5.10). (For the latter equation, multiply by $\exp \left\{-\eta_{1}(\xi) x\right\}$ and integrate over the interval $[0, K]$.) Finally, determine $Q$ and $\mathrm{P}(Z<K)$. Letting $\omega \downarrow 0$ in (5.3) and applying l'Hôpital's rule gives

$$
Q=\phi_{2}^{\prime}(0)-\left(\phi_{2}^{\prime}(0)-\phi_{1}^{\prime}(0)\right) \mathrm{P}(Z<K),
$$


and substituting $x=K$ into (5.10) gives

$$
\mathrm{P}(Z<K)=Q W_{1}(K)+\left(r_{1}-r_{2}\right) P_{\downarrow K} \int_{0}^{K} W_{2}^{(T)}(K-y ; K) \mathrm{d} W_{1}(y) .
$$

\section{2. $\mathrm{M} / \mathrm{G} / 1$ queues}

In this subsection we assume that $\phi_{i}(\omega)=r_{i} \omega-\lambda_{i}+\lambda_{i} \beta_{i}(\omega)$ for $i=1,2$. Recall that the stability condition reads $\rho_{2}<1$, with $\rho_{i}=\lambda_{i} \beta_{i} / r_{i}, i=1$, 2 . In this case, the scale function $W_{i}(\cdot)$ can be explicitly determined (for $\rho_{1} \geq 1$, the explicit form is involved).

To describe the scale function $W_{i}(\cdot)$, we define

$$
H_{i}(x):=\beta_{i}^{-1} \int_{0}^{x}\left(1-B_{i}(y)\right) \mathrm{d} y
$$

as the stationary residual service requirement distribution of a generic service requirement $B_{i}, i=1,2$. In the case in which $\rho_{i}<1$, it is well known that

$$
W_{i}(x)=\frac{1}{r_{i}} \sum_{n=0}^{\infty} \rho_{i}^{n} H_{i}^{n^{*}}(x),
$$

which is directly related to the steady-state workload distribution in an M/G/1 queue. In fact, it may be checked that the LST of $\left(1-\rho_{i}\right) r_{i} W_{i}(\cdot)$ equals

$$
\mathrm{E}\left[\mathrm{e}^{-\omega Z}\right]=\frac{\left(1-\rho_{i}\right) r_{i} \omega}{r_{i} \omega-\lambda_{i}+\lambda_{i} \beta_{i}(\omega)},
$$

which is given by Theorem 2.1. Since we assumed that $\rho_{2}<1$ for stability, this provides $W_{2}(\cdot)$. However, we allow $\rho_{1} \geq 1$. To obtain $W_{1}(\cdot)$, we apply the arguments of [13] and [12]. Let $\delta_{1}=0$ for $\rho_{1} \leq 1$ and, for $\rho_{1}>1$, let $\delta_{1}$ be the unique positive zero of the function

$$
\int_{0}^{\infty} \mathrm{e}^{-x y} \rho_{1} \mathrm{~d} H_{1}(y)-1 .
$$

Then, for $x>0$, define

$$
L(x):=\int_{0}^{x} \exp \left\{-\delta_{1} y\right\} \rho_{1} \mathrm{~d} H_{1}(y) \quad \text { and } \quad W_{1}(x):=\frac{1}{r_{1}} \int_{0^{-}}^{x} \exp \left\{\delta_{1} y\right\} \mathrm{d}\left\{\sum_{n=0}^{\infty} L^{n^{*}}(y)\right\},
$$

where $L^{n^{*}}(\cdot)$ denotes the $n$-fold convolution of $L(\cdot)$ with itself. It may be checked that, as in [12] and [13], the Laplace transform of $W_{1}(\cdot)$ equals $1 / \phi_{1}(\omega)$.

In the case in which $\rho_{1} \geq 1, W_{1}(\cdot)$ may be interpreted in terms of a dam with release rate $r_{1}$ and capacity $K$. Specifically, the stationary workload distribution for such a dam equals $W_{1}(\cdot) / W_{1}(K)$; see, e.g. [12], [14, p. 536], or (2.3).

The special case in which only the service speed is adapted can be directly obtained from Subsection 5.1 and the explicit form of the scale function. Moreover, the results become especially tractable in the case where the service requirements have an exponential distribution function. A probabilistic approach for this $\mathrm{M} / \mathrm{M} / 1$ model can be found in [5]. In the general M/G/1 setting, the steady-state workload distribution can be obtained from (5.3) and (5.8), and by rewriting the fraction of Lévy exponents as in Subsection 4.2. Because of similarities with Subsections 4.2 and 5.1, we only give an outline. 
Step 2: Workload distribution on $(0, K]$. Again, we apply Laplace inversion to each of the three terms on the right-hand side of (5.8). The inverse of the first term is given by $Q W_{1}(\cdot)$; see Definition 2.1. The second term corresponds to the LST of a function with mass only on $[K, \infty)$. To see this, we note that $\int_{K}^{\infty} \mathrm{E}\left[\exp \left\{-\omega Z_{2}^{(T)}\right\} \mathbf{1}\left(T<\tau_{K}\right) \mid Z_{2}^{(0)}=x\right] \operatorname{dP}(Z<x)$ is the transform of a function with mass on $[K, \infty)$, while the term $\left(\phi_{1}(\omega)-\phi_{2}(\omega)\right) / \phi_{1}(\omega)$ can be treated as in Subsection 4.2.

For the third term on the right-hand side of (5.8), we note that $\mathrm{E}\left[\exp \left\{-\omega Z_{2}^{(T)}\right\} \mid Z_{2}^{(0)}=K\right]$ is the LST of $W^{(T)}(\cdot ; K)$, corresponding to the amount of work after an exponential time starting from $K$. Rewriting the term $\left(\phi_{1}(\omega)-\phi_{2}(\omega)\right) / \phi_{1}(\omega)$ again, and applying similar arguments as in Subsection 4.2, we may invert the third term. Specifically, let $W_{1}^{\text {exc }}(\cdot)$ be the steady-state workload distribution in an M/G/1 queue with service rate $r_{1}$, arrival rate $\lambda_{1}$, and generic service requirement $B_{1}$, but with exceptional first service $B_{2}$ in a busy period (see, e.g. [35], [36], or Example A.1). For simplicity, we assume here that $\rho_{1}<1$. Applying Laplace inversion then yields, for $x \in(0, K]$,

$$
\begin{aligned}
\mathrm{P}(Z<x)=Q W_{1}(x)+P_{\downarrow K} & \left(\frac{\lambda_{2}}{\lambda_{1}} \frac{1+\lambda_{1} \beta_{2} / r_{1}-\rho_{1}}{1-\rho_{1}} \int_{0}^{x} W^{(T)}(x-y ; K) \mathrm{d} W_{1}^{\text {exc }}(y)\right. \\
& \left.-r_{2} \int_{0}^{x} W^{(T)}(x-y ; K) \mathrm{d} W_{1}(y)+\left(1-\frac{\lambda_{2}}{\lambda_{1}}\right) W^{(T)}(x ; K)\right) .
\end{aligned}
$$

Step 3: Workload distribution on $(K, \infty)$. Apply Laplace inversion to each of the terms on the right-hand side of (5.3). The Laplace inverse of the first term is given by $Q W_{2}(\cdot)$. For the second term on the right-hand side of (5.3), it follows from Theorem 2.2 that

$$
\frac{\xi}{\xi-\phi_{1}(\omega)}\left(\int_{0}^{K} \mathrm{e}^{-\omega x} \mathrm{dP}(Z<x)-\omega \int_{0}^{K} \frac{\exp \left\{-\eta_{1}(\xi) x\right\}}{\eta_{1}(\xi)} \mathrm{dP}(Z<x)\right)
$$

is the LST of $\tilde{W}^{(T)}(\cdot)$, i.e. the amount of work of a reflected Lévy process with exponent $\phi_{1}(\omega)$ after an exponential time, starting according to $\mathrm{P}(Z<x)$. Since we have determined the distribution of $Z$ on $[0, K]$, this also formally gives $\tilde{W}^{(T)}(\cdot)$ (although the precise form is again rather involved).

Now, rewriting the term $\left(\phi_{2}(\omega)-\phi_{1}(\omega)\right) / \phi_{2}(\omega)$ similarly as in Subsection 4.2, we can invert each term separately. Recall that $W_{2}^{\text {exc }}(\cdot)$ denotes the workload distribution in an M/G/1 queue with service rate $r_{2}$, arrival rate $\lambda_{2}$, and generic service requirement $B_{2}$, but with exceptional first service $B_{1}$ in a busy period. Applying similar arguments as in Subsections 4.2 and 5.1, we find, by Laplace inversion, that, for $x>K$,

$$
\begin{aligned}
\mathrm{P}(Z<x)= & Q W_{2}(x)+\frac{\lambda_{1}}{\lambda_{2}} \frac{1+\lambda_{2} \beta_{1} / r_{2}-\rho_{2}}{1-\rho_{2}} \int_{0}^{x} W_{2}^{\mathrm{exc}}(x-y) \mathrm{d} \tilde{W}^{(T)}(y) \\
& -r_{1} \int_{0}^{x} W_{2}(x-y) \mathrm{d} \tilde{W}^{(T)}(y)+\left(1-\frac{\lambda_{1}}{\lambda_{2}}\right) \tilde{W}^{(T)}(x) .
\end{aligned}
$$

Step 4: Determination of the constants. The constants can be determined similarly as in Subsection 5.1; see also [4].

\subsection{Brownian motion}

In this subsection we consider the special case of Brownian motion, i.e. we assume that $\phi_{i}(\omega)=\omega^{2} \sigma_{i}^{2} / 2-\mu_{i} \omega, i=1,2$. For stability, we require that $\mu_{2}<0$. In the case of Brownian motion, the steady-state workload distribution has a rather tractable form. 
First, consider the $q$-scale function $W_{i}^{(q)}(\cdot)$. Define $\delta_{i}(q):=\sqrt{\mu_{i}^{2}+2 q \sigma_{i}^{2}}$, and let $\eta_{i}^{ \pm}(q)$ be the positive and negative solutions, respectively, of $\phi_{i}(\omega)-q$, that is,

$$
\eta_{i}^{ \pm}(q):=\frac{\mu_{i} \pm \delta_{i}(q)}{\sigma_{i}^{2}}=\frac{\mu_{i} \pm \sqrt{\mu_{i}^{2}+2 q \sigma_{i}^{2}}}{\sigma_{i}^{2}} .
$$

We note that $\eta_{i}^{+}(q)$ equals $\eta_{i}(q)$, as defined in Theorem 2.2. The $q$-scale function now reads

$$
W_{i}^{(q)}(x)=\frac{1}{\delta_{i}(q)}\left(\exp \left\{\eta_{i}^{+}(q) x\right\}-\exp \left\{\eta_{i}^{-}(q) x\right\}\right)
$$

see also [27] for the spectrally negative case with $\sigma_{i}=1$. Given Theorem 2.1, of special interest is the 0 -scale function. In the case in which $\mu_{i}<0$, we have $W_{i}(x)=\left(1-\exp \left\{2 \mu_{i} x / \sigma_{i}^{2}\right\}\right) /\left|\mu_{i}\right|$, and (2.2) indeed reduces to the familiar steady-state distribution of a reflected Brownian motion.

Finally, the density of the amount of work after an exponential time starting from $v$ follows from the derivative with respect to $x$ of (2.5), or can be obtained from Laplace inversion in Theorem 2.2. Specifically, for $x \in(0, v)$, we have

$$
\frac{\mathrm{d}}{\mathrm{d} x} W_{i}^{(T)}(x ; v)=\frac{\xi}{\delta_{i}(\xi)} \exp \left\{-\eta_{i}^{+}(\xi) v\right\}\left(\exp \left\{\eta_{i}^{+}(\xi) x\right\}-\frac{\eta_{i}^{-}(\xi)}{\eta_{i}^{+}(\xi)} \exp \left\{\eta_{i}^{-}(\xi) x\right\}\right),
$$

and, for $x \in[v, \infty)$, we have

$$
\frac{\mathrm{d}}{\mathrm{d} x} W_{i}^{(T)}(x ; v)=\frac{\xi}{\delta_{i}(\xi)}\left(\exp \left\{-\eta_{i}^{-}(\xi) v\right\}-\frac{\eta_{i}^{-}(\xi)}{\eta_{i}^{+}(\xi)} \exp \left\{-\eta_{i}^{+}(\xi) v\right\}\right) \exp \left\{\eta_{i}^{-}(\xi) x\right\}
$$

Step 1: Rewriting (5.8). Note that

$$
\frac{\phi_{1}(\omega)-\phi_{2}(\omega)}{\phi_{1}(\omega)}=\frac{\mu_{2}-\mu_{1}+(1 / 2)\left(\sigma_{1}^{2}-\sigma_{2}^{2}\right) \omega}{\sigma_{1}^{2} \omega / 2-\mu_{1}}=1-\frac{\sigma_{2}^{2}}{\sigma_{1}^{2}}+\left(\frac{\mu_{2}}{\mu_{1}}-\frac{\sigma_{2}^{2}}{\sigma_{1}^{2}}\right) \frac{2 \mu_{1} / \sigma_{1}^{2}}{\omega-2 \mu_{1} / \sigma_{1}^{2}}
$$

Substitution into (5.8) then gives

$$
\begin{aligned}
\zeta(\omega)=Q \frac{\omega}{\phi_{1}(\omega)}+ & \left(1-\frac{\sigma_{2}^{2}}{\sigma_{1}^{2}}+\left(\frac{\mu_{2}}{\mu_{1}}-\frac{\sigma_{2}^{2}}{\sigma_{1}^{2}}\right) \frac{2 \mu_{1} / \sigma_{1}^{2}}{\omega-2 \mu_{1} / \sigma_{1}^{2}}\right) \\
& \times\left(\int_{K}^{\infty} \mathrm{E}\left[\exp \left\{-\omega Z_{2}^{(T)}\right\} \mathbf{1}\left(T<\tau_{K}\right) \mid Z_{2}^{(0)}=x\right] \mathrm{dP}(Z<x)\right. \\
& \left.+P_{\downarrow K} \mathrm{E}\left[\exp \left\{-\omega Z_{2}^{(T)}\right\} \mid Z_{2}^{(0)}=K\right]\right) .
\end{aligned}
$$

Step 2: Workload density on $(0, K]$. Apply Laplace inversion to each of the terms on the right-hand side of (5.14) separately. The Laplace inverse of the first term is simply given by a constant times the scale function $W_{1}(\cdot)$. The second term,

$$
\left(1-\frac{\sigma_{2}^{2}}{\sigma_{1}^{2}}+\left(\frac{\mu_{2}}{\mu_{1}}-\frac{\sigma_{2}^{2}}{\sigma_{1}^{2}}\right) \frac{2 \mu_{1} / \sigma_{1}^{2}}{\omega-2 \mu_{1} / \sigma_{1}^{2}}\right) \int_{K}^{\infty} \mathrm{E}\left[\exp \left\{-\omega Z_{2}^{(T)}\right\} \mathbf{1}\left(T<\tau_{K}\right) \mid Z_{2}^{(0)}=x\right] \operatorname{dP}(Z<x),
$$

is the LST of a function that has no mass on $[0, K]$. Indeed, the integral corresponds to a function with mass on $(K, \infty)$. The first part consists of a constant and the LST of the scale function $W_{1}(\cdot)$ (having mass on $[0, \infty)$ ) times a constant. 
Using (5.11) and the fact that a product of two transforms corresponds to the convolution of two functions, it is a matter of tedious calculations to determine the Laplace inverse of the final term. Denote by $f_{Z}(\cdot)$ the density of $Z$. For $0<x \leq K$, we then have

$$
f_{Z}(x)=Q_{1} \exp \left\{\frac{2 \mu_{1} x}{\sigma_{1}^{2}}\right\}+Q_{2} \exp \left\{\eta_{2}^{+}(\xi) x\right\}+Q_{3} \exp \left\{\eta_{2}^{-}(\xi) x\right\}
$$

for some constants $Q_{1}, Q_{2}$, and $Q_{3}$.

Step 3: Workload density on $(K, \infty)$. Combining step 2 with (5.3) gives the $\operatorname{LST} \zeta(\omega)$. The density $f_{Z}(\cdot)$ on $(K, \infty)$ can be determined by applying Laplace inversion again. Using Theorem 2.2 and a similar calculation as in (5.13), we may rewrite (5.3) as

$$
\begin{aligned}
\zeta(\omega)=Q \frac{\omega}{\phi_{2}(\omega)}+ & \left(1-\frac{\sigma_{1}^{2}}{\sigma_{2}^{2}}+\left(\frac{\mu_{1}}{\mu_{2}}-\frac{\sigma_{1}^{2}}{\sigma_{2}^{2}}\right) \frac{2 \mu_{2} / \sigma_{2}^{2}}{\omega-2 \mu_{2} / \sigma_{2}^{2}}\right) \\
& \times \int_{0}^{K} \mathrm{E}\left[\exp \left\{-\omega Z_{1}^{(T)}\right\} \mid Z_{1}^{(0)}=y\right] f_{Z}(y) \mathrm{d} y .
\end{aligned}
$$

Using (5.15) of step 2 combined with (5.11) and (5.12), we may determine the distribution function $\tilde{W}^{(T)}(\cdot)$ (and its density). However, it turns out to be sufficient to determine the density $\tilde{W}^{(T)}(\in \mathrm{d} x)$ for only $x \geq K$; see also step 4, below. In this case, it follows, from (5.12) and (5.15) and some straightforward algebra, that, for $x>K$,

$$
\frac{\mathrm{d}}{\mathrm{d} x} \tilde{W}^{(T)}(x)=\tilde{Q} \exp \left\{\eta_{1}^{-}(\xi) x\right\}
$$

Now, applying Laplace inversion to each of the terms in (5.16) we obtain, after some calculations for $x \geq K$, and for some constants $Q_{4}$ and $Q_{5}$,

$$
f_{Z}(x)=Q_{4} \exp \left\{\eta_{1}^{-}(\xi) x\right\}+Q_{5} \exp \left\{\frac{2 \mu_{2} x}{\sigma_{2}^{2}}\right\} .
$$

Step 4: Determination of the constants $Q_{i}, i=1, \ldots, 5$. We note that, for the case of Brownian motion, there is no atom at 0 (to see this, observe that $W_{i}^{(q)}(0)=0$ ). First, we have the normalizing condition $\int_{0}^{\infty} f_{Z}(x) \mathrm{d} x=1$. The lengthy calculations to determine $Q_{i}, i=1, \ldots, 4$, in steps 2 and 3 also provide us with four equations. Together, we then have five independent equations to determine the five unknowns $Q_{i}, i=1, \ldots, 5$. Since we also find the constant $Q_{5}$, there is no need to specify $Q_{5}$ any further in step 3 .

\section{Poisson observer: general solution}

Now consider the general solution of the reflected Lévy process where the Lévy exponent is adapted at Poisson instants. Since it is in general not possible to reduce $\left(\phi_{i}(\omega)-\right.$ $\left.\phi_{3-i}(\omega)\right) / \phi_{i}(\omega), i=1,2$, we use a slightly different approach in this section than in Section 5 . In particular, in step 0 we use the Palm inversion formula and results on alternating Lévy processes as defined in Appendix A to obtain two sets of equations for $\zeta(\cdot)$. The interpretation of the specific alternating Lévy processes allows us to carry out steps 1-4 in the general case. We believe that the more direct and insightful derivation in Section 5 is of independent interest, leading to more tractable expressions for $\mathrm{P}(Z<x)$.

In step 0 we derive a first set of equations using a specific alternating Lévy process. Since we apply a different alternating Lévy process for the second set of equations, we have chosen to derive this second set of equations in step 3 , where the specific alternating process is introduced. 
Step 0: Determining the equations. Assume that the process is in stationarity and consider the workload embedded at epochs when the Poisson observer sees a workload larger than $K$. To describe the workload behavior in periods between these embedded epochs, we introduce the following artificial regenerative alternating Lévy process $\left\{\hat{Z}^{(t)}, t \geq 0\right\}$. Let the process $\hat{Z}^{(t)}$ start from some level $x \geq K$ according to $\mathrm{P}(Z<x \mid Z \geq K)$, independent of the past evolution, and define $\theta_{1}=T$, the first observer epoch after 0 . During this first period, the Lévy exponent is taken to be $\phi_{2}(\cdot)$. At time $\theta_{1}$, the Lévy exponent is changed into $\phi_{1}(\cdot)$. We define $\theta_{2}>0$ as the first observer instant after time 0 with a workload larger than or equal to $K$. It should be noted that it is possible that $\theta_{2}$ coincides with $\theta_{1}$. Then the period during which the Lévy exponent equals $\phi_{1}(\cdot)$ has length 0 . Times 0 and $\theta_{2}$ are two consecutive observer epochs with a workload larger than $K$. Owing to stationarity, we have $\mathrm{E}\left[\exp \left\{-\omega \hat{Z}^{(0)}\right\}\right]=\mathrm{E}\left[\exp \left\{-\omega \hat{Z}^{\left(\theta_{2}\right)}\right\}\right]$. Applying Lemma A.1 we obtain

$\mathrm{E}\left[\mathrm{e}^{-\omega \hat{Z}}\right]=\frac{\mathrm{E}\left[\hat{L}_{2}^{\left(\theta_{2}-\theta_{1}\right)}\right]+\mathrm{E}\left[\hat{L}_{1}^{\left(\theta_{1}\right)}\right]}{\mathrm{E}\left[\theta_{2}\right]} \frac{\omega}{\phi_{1}(\omega)}+\frac{\phi_{1}(\omega)-\phi_{2}(\omega)}{\phi_{1}(\omega)} \frac{1}{\mathrm{E}\left[\theta_{2}\right]} \mathrm{E}\left[\int_{s=0}^{\theta_{1}} \exp \left\{-\omega \hat{Z}^{(s)}\right\} \mathrm{d} s\right]$.

In fact, the LST of the actual process $Z$ and the regenerative process $\hat{Z}$ are identical. To see this, we consider the observer instants with a workload larger than or equal to $K$ of the actual process $Z$ (i.e. the embedded epochs) as event times and apply the Palm inversion formula [3], [33]:

$$
\zeta(\omega)=\frac{1}{\mathrm{E}\left[\theta_{2}\right]} \mathrm{E}\left[\int_{0}^{\theta_{2}} \exp \left\{-\omega Z^{(s)}\right\} \mathrm{d} s\right]=\frac{1}{\mathrm{E}\left[\theta_{2}\right]} \mathrm{E}\left[\int_{0}^{\theta_{2}} \exp \left\{-\omega \hat{Z}^{(s)}\right\} \mathrm{d} s\right]=\mathrm{E}\left[\mathrm{e}^{-\omega \hat{Z}}\right] .
$$

Here, the second step is by construction of the process $\hat{Z}$ and the third step follows from regeneration theory. Thus, (6.1) gives a first set of equations for the LST of $Z$.

Remark 6.1. Note that (5.2) and (6.1) are identical. Write, for $x \geq K, \mathrm{P}(Z<x)=\mathrm{P}(Z<$ $K)+\mathrm{P}(Z<x \mid Z \geq K) \mathrm{P}(Z \geq K)$, and let $x$ in $\mathrm{E}_{x}[\cdot]$ denote the initial position of the process. Then,

$$
\begin{aligned}
\mathrm{E}\left[\int_{0}^{\theta_{1}} \exp \left\{-\omega \hat{Z}^{(s)}\right\} \mathrm{d} s\right] & =\int_{K}^{\infty} \mathrm{E}_{x}\left[\int_{0}^{\theta_{1}} \exp \left\{-\omega \hat{Z}^{(s)}\right\} \mathrm{d} s\right] \mathrm{dP}(Z<x \mid Z \geq K) \\
& =\frac{1}{\mathrm{P}(Z \geq K)} \int_{K}^{\infty} \mathrm{E}_{x}\left[\int_{0}^{\theta_{1}} \exp \left\{-\omega \hat{Z}^{(s)}\right\} \mathrm{d} s\right] \mathrm{dP}(Z<x) \\
& =\frac{\mathrm{E}\left[\theta_{1}\right]}{\mathrm{P}(Z \geq K)} \int_{K}^{\infty} \mathrm{E}_{x}\left[\exp \left\{-\omega Z_{2}^{(T)}\right\}\right] \mathrm{dP}(Z<x)
\end{aligned}
$$

where the final step follows from (2.4). The fraction of time that the Lévy exponent is $\phi_{2}(\cdot)$ equals the fraction of observers finding a workload larger than $K$, and, hence, using the PASTA property, also equals the fraction of time that the workload is larger than $K$. An application of the Palm inversion formula then provides $\mathrm{P}(Z \geq K)=\mathrm{E}\left[\theta_{1}\right] / \mathrm{E}\left[\theta_{2}\right]$. Combining the above and using Theorem 2.2, it follows that the second terms on the right-hand sides of (5.2) and (6.1) are identical, and (6.1) can be rewritten as (3.2).

For the first terms, we have

$$
\frac{\mathrm{E}\left[\hat{L}_{2}^{\left(\theta_{2}-\theta_{1}\right)}\right]+\mathrm{E}\left[\hat{L}_{1}^{\left(\theta_{1}\right)}\right]}{\mathrm{E}\left[\theta_{2}\right]}=\lim _{t \rightarrow \infty} \frac{\mathrm{E}\left[L^{(t)}\right]}{t}=Q,
$$


where the first step follows from an application of the Palm inversion formula and the second step is due to starting in stationarity.

Step 1: Rewriting (6.1). Use the fact that periods with Lévy exponent $\phi_{2}(\cdot)$ and workloads smaller than $K$ are initiated by an observer finding a workload larger than $K$ (to set the exponent to $\left.\phi_{2}(\cdot)\right)$ followed by a downcrossing of level $K$ before the next observer instant. Owing to the lack-of-memory property of the Poisson arrival process, the remaining interobservation time is still exponential at a downcrossing of $K$. Using the stationary and independent increments property of Lévy processes, it is intuitively clear that the precise distribution of $Z$ on $(K, \infty)$ affects only the distribution of $Z$ on $[0, K]$ through a constant.

The above intuition can be applied as follows. Let $\mathrm{E}_{Z \geq K}\left[\mathrm{e}^{-\omega \hat{Z}}\right]$ for $\int_{K}^{\infty} \mathrm{E}_{x}\left[\mathrm{e}^{-\omega \hat{Z}}\right] \mathrm{dP}(Z<$ $x \mid Z \geq K$ ). Define, with some abuse of notation (see already the hitting time $\tau_{K}$ in Section 5), $\tau_{K}:=\inf \left[t \geq 0: \hat{Z}^{(t)}=K\right]$ as the first hitting time of $K$, and let $\mathbf{1}(\cdot)$ again denote the indicator function. Now, using (2.4) in the second and fourth equalities below, and the lack-of-memory property of the Poisson arrival process and the Lévy process in the third equality, we have

$$
\begin{aligned}
\mathrm{E}_{Z \geq K}\left[\mathrm{e}^{-\omega \hat{Z}}\right]= & \frac{1}{\mathrm{E}\left[\theta_{2}\right]} \mathrm{E}_{Z \geq K}\left[\int_{0}^{\theta_{2}} \exp \left\{-\omega \hat{Z}^{(s)}\right\} \mathbf{1}\left(T<\tau_{K}\right) \mathrm{d} s\right] \\
& +\frac{1}{\mathrm{E}\left[\theta_{2}\right]} \mathrm{E}_{Z \geq K}\left[\int_{0}^{\theta_{2}} \exp \left\{-\omega \hat{Z}^{(s)}\right\} \mathbf{1}\left(T \geq \tau_{K}\right) \mathrm{d} s\right] \\
= & \frac{1}{\mathrm{E}\left[\theta_{2}\right]} \mathrm{E}_{Z \geq K}\left[\int_{0}^{T} \exp \left\{-\omega \hat{Z}^{(s)}\right\} \mathbf{1}\left(T<\tau_{K}\right) \mathrm{d} s\right] \\
& +\frac{1}{\xi \mathrm{E}\left[\theta_{2}\right]} \mathrm{E}_{Z \geq K}\left[\exp \left\{-\omega \hat{Z}^{(T)}\right\} \mathbf{1}\left(T \geq \tau_{K}\right) \mathrm{d} s\right] \\
& +\frac{1}{\mathrm{E}\left[\theta_{2}\right]} \mathrm{E}_{Z \geq K}\left[\int_{\theta_{1}}^{\theta_{2}} \exp \left\{-\omega \hat{Z}^{(s)}\right\} \mathbf{1}\left(T \geq \tau_{K}\right) \mathrm{d} s\right] \\
= & \frac{1}{\mathrm{E}\left[\theta_{2}\right]} \mathrm{E}_{Z \geq K}\left[\int_{0}^{T} \exp \left\{-\omega \hat{Z}^{(s)}\right\} \mathbf{1}\left(T<\tau_{K}\right) \mathrm{d} s\right] \\
& +\frac{1}{\left.\xi \mathrm{E}_{2} \theta_{2}\right]} \hat{P}_{\downarrow K} \mathrm{E}_{K}\left[\exp \left\{-\omega \hat{Z}^{(T)}\right\} \mathrm{d} s\right] \\
& +\frac{1}{\mathrm{E}\left[\theta_{2}\right]} \hat{P}_{\downarrow K} \mathrm{E}_{K}\left[\int_{\theta_{1}}^{\theta_{2}} \exp \left\{-\omega \hat{Z}^{(s)}\right\} \mathrm{d} s\right] \\
= & \frac{1}{\mathrm{E}\left[\theta_{2}\right]} \mathrm{E}_{Z \geq K}\left[\int_{0}^{T} \exp \left\{-\omega \hat{Z}^{(s)}\right\} \mathbf{1}\left(T<\tau_{K}\right) \mathrm{d} s\right]+\hat{P}_{\downarrow K} \frac{\mathrm{E}_{K}\left[\theta_{2}\right]}{\mathrm{E}\left[\theta_{2}\right]} \mathrm{E}_{K}\left[\mathrm{e}^{-\omega \hat{Z}}\right],
\end{aligned}
$$

where $\hat{P}_{\downarrow K}$ is the probability of downcrossing $K$ before an exponential time with parameter $\xi$, starting from $x$ according to $\mathrm{P}(Z<x \mid Z \geq K)$. Using results on the LST of first-exit times (see, e.g. [24]), it may be checked that

$$
\begin{aligned}
\hat{P}_{\downarrow K} & =\xi \exp \left\{\eta_{2}(\xi) K\right\} \int_{K}^{\infty} \exp \left\{-\eta_{2}(\xi) x\right\} \mathrm{dP}(Z<x \mid Z \geq K) \\
& =\frac{\xi}{\mathrm{P}(Z \geq K)} \exp \left\{\eta_{2}(\xi) K\right\} \int_{K}^{\infty} \exp \left\{-\eta_{2}(\xi) x\right\} \mathrm{dP}(Z<x) .
\end{aligned}
$$


The first transform on the last line of (6.2) gives no contribution on $[0, K]$, since $\hat{Z}^{(s)}>K$ for all $s \in[0, T]$. Thus, the distribution of $\hat{Z}$, and, hence, that of $Z$, on the interval $[0, K]$ is completely determined by the second transform $\mathrm{E}_{K}\left[\mathrm{e}^{-\omega \hat{Z}}\right]$, where the initial condition depends only on the distribution of $Z$ on $[K, \infty)$ through the constant $\hat{P}_{\downarrow K} \mathrm{E}_{K}\left[\theta_{2}\right] / \mathrm{E}\left[\theta_{2}\right]$.

Step 2: Workload distribution on $(0, K]$. Apply Laplace inversion to $\mathrm{E}_{K}\left[\mathrm{e}^{-\omega \hat{Z}}\right]$. Using Lemma A.2, this transform satisfies

$$
\begin{aligned}
\mathrm{E}_{K}\left[\mathrm{e}^{-\omega \hat{Z}}\right]= & \frac{\mathrm{E}\left[\hat{L}_{2}^{\left(\theta_{2}-\theta_{1}\right)}\right]}{\mathrm{E}_{K}\left[\theta_{2}\right]} \frac{\omega}{\phi_{1}(\omega)}+\frac{1}{\mathrm{E}_{K}\left[\theta_{2}\right]} \frac{\mathrm{E}\left[\exp \left\{-\omega \hat{Z}^{\left(\theta_{2}\right)}\right\}\right]-\mathrm{E}\left[\exp \left\{-\omega \hat{Z}^{\left(\theta_{1}\right)}\right\}\right]}{\phi_{1}(\omega)} \\
& +\frac{1}{\mathrm{E}_{K}\left[\theta_{2}\right]} \mathrm{E}\left[\int_{s=0}^{\theta_{1}} \exp \left\{-\omega \hat{Z}^{(s)}\right\} \mathrm{d} s\right] .
\end{aligned}
$$

In this step, the initial position of the process is $K$. For notational convenience, we suppress this initial position, except for $\mathrm{E}_{K}\left[\theta_{2}\right]$ (to distinguish from $\mathrm{E}\left[\theta_{2}\right]$ ).

Using Theorem 2.1, (2.4), and Theorem 2.2, we directly obtain the inverse of the first and third terms on the right-hand side of the above equation as constants times $W_{1}(\cdot)$ and $W_{2}^{(T)}(\cdot ; K)$, respectively. It remains to find the Laplace inverse on $(0, K]$ of the second term.

Observe that $\theta_{1}=\theta_{2}$ if and only if $\hat{Z}^{\left(\theta_{1}\right)} \geq K$. Thus, we may write

$$
\begin{aligned}
& \mathrm{E}\left[\exp \left\{-\omega \hat{Z}^{\left(\theta_{2}\right)}\right\}\right]-\mathrm{E}\left[\exp \left\{-\omega \hat{Z}^{\left(\theta_{1}\right)}\right\}\right] \\
& \quad=\mathrm{E}\left[\exp \left\{-\omega \hat{Z}^{\left(\theta_{2}\right)}\right\} \mathbf{1}\left(\hat{Z}^{\left(\theta_{1}\right)}<K\right)\right]-\mathrm{E}\left[\exp \left\{-\omega \hat{Z}^{\left(\theta_{1}\right)}\right\} \mathbf{1}\left(\hat{Z}^{\left(\theta_{1}\right)}<K\right)\right] \\
& \quad=\mathrm{P}\left(\hat{Z}^{\left(\theta_{1}\right)}<K\right)\left(\mathrm{E}\left[\exp \left\{-\omega \hat{Z}^{\left(\theta_{2}\right)}\right\} \mid \theta_{2}>\theta_{1}\right]-\mathrm{E}\left[\exp \left\{-\omega \hat{Z}^{\left(\theta_{1}\right)}\right\} \mid \hat{Z}^{\left(\theta_{1}\right)}<K\right]\right) \\
& \quad=\mathrm{P}\left(\hat{Z}^{\left(\theta_{1}\right)}<K\right) \mathrm{e}^{-\omega K}\left(\mathrm{E}\left[\exp \left\{-\omega\left(\hat{Z}^{\left(\theta_{2}\right)}-K\right)\right\} \mid \theta_{2}>\theta_{1}\right]-1+1-\mathrm{E}\left[\mathrm{e}^{\omega U} \mid U>0\right]\right),
\end{aligned}
$$

where $U:=K-\hat{Z}^{\left(\theta_{1}\right)}$ is the undershoot under $K$ at the end of the first exponential time $\theta_{1}$ starting from level $K$. For the second term on the right-hand side of (6.4), we then have

$$
\begin{aligned}
\frac{\mathrm{E}\left[\exp \left\{-\omega \hat{Z}^{\left(\theta_{2}\right)}\right\}\right]-\mathrm{E}\left[\exp \left\{-\omega \hat{Z}^{\left(\theta_{1}\right)}\right\}\right]}{\phi_{1}(\omega)} \\
=-\mathrm{P}\left(\hat{Z}^{\left(\theta_{1}\right)}<K\right) \frac{\omega}{\phi_{1}(\omega)} \mathrm{e}^{-\omega K} \frac{1-\mathrm{E}\left[\exp \left\{-\omega\left(\hat{Z}^{\left(\theta_{2}\right)}-K\right)\right\} \mid \theta_{2}>\theta_{1}\right]}{\omega} \\
\quad-\mathrm{P}\left(\hat{Z}^{\left(\theta_{1}\right)}<K\right) \frac{\omega}{\phi_{1}(\omega)} \mathrm{e}^{-\omega K} \frac{1-\mathrm{E}[\exp \{\omega U\} \mid U>0]}{-\omega} .
\end{aligned}
$$

For the first term on the right-hand side of the above equation, we note that $\hat{Z}^{\left(\theta_{2}\right)}-K \geq 0$ corresponds to the overshoot over $K$. Hence, $\mathrm{e}^{-\omega K}\left(1-\mathrm{E}\left[\exp \left\{-\omega\left(\hat{Z}^{\left(\theta_{2}\right)}-K\right)\right\} \mid \theta_{2}>\theta_{1}\right]\right) / \omega$ corresponds to the sum of the constant $K$ and the residual overshoot at time $\theta_{2}$, given that $\theta_{2}>\theta_{1}$. Clearly, the corresponding convolution has no mass on $[0, K]$. Since $\omega / \phi_{1}(\omega)$ has mass on $[0, \infty)$, this first term corresponds to the LST of a function with mass on $(K, \infty)$.

The second term can be interpreted as the transform of a sum as well. First note that the conditional undershoot $U \mid U>0$ has mass on $[0, K]$. The transform $\mathrm{e}^{-\omega K}\left(1-\mathrm{E}\left[\mathrm{e}^{\omega U} \mid U>\right.\right.$ $0]) /(-\omega \mathrm{E}[U \mid U>0])$ then corresponds to $K-U^{\text {res }}$, where $U^{\text {res }}$ represents a generic residual undershoot given that $U>0$. Using Theorem 2.1 and Definition 2.1, the Laplace inverse of the second term reads

$$
W_{1}(x) * \mathrm{P}\left(K-U^{\text {res }} \leq x \mid U>0\right) \mathrm{E}[U \mid U>0] .
$$


Summarizing, we have, for $x \in(0, K]$,

$$
\begin{aligned}
& \mathrm{P}(Z<x) \\
& \begin{aligned}
=\frac{\hat{P}_{\downarrow K}}{\mathrm{E}\left[\theta_{2}\right]}\left(\mathrm{E}\left[\hat{L}_{2}^{\left(\theta_{2}-\theta_{1}\right)}\right] W_{1}(x)+\frac{1}{\xi} W_{2}^{(T)}(x ; K)\right. \\
\left.\quad-W_{2}^{(T)}(K ; K) \mathrm{E}[U \mid U>0] \int_{0}^{x} \mathrm{P}\left(K-U^{\text {res }}<x-y \mid U>0\right) \mathrm{d} W_{1}(y)\right) .
\end{aligned}
\end{aligned}
$$

Although the distribution of $K-U^{\text {res }}$ may be rather involved, we note that $\mathrm{P}(Z<x)$ for $x \in(0, K]$ depends only on the distribution of $Z$ on $(K, \infty)$ through a constant.

Remark 6.2. We may also give another representation of the Laplace inverse of the second term on the right-hand side of (6.4). Using Theorem 2.1 and Definition 2.1, we easily find that $1 / \phi_{1}(\omega)$ is the LST of $\int_{0}^{x} W_{1}(y) \mathrm{d} y$. Clearly, this function has mass on $[0, \infty)$. Rewriting the second term on the right-hand side of (6.4), we obtain

$\frac{\mathrm{E}\left[\exp \left\{-\omega \hat{Z}^{\left(\theta_{2}\right)}\right\}\right]-\mathrm{E}\left[\exp \left\{-\omega \hat{Z}^{\left(\theta_{1}\right)}\right\}\right]}{\phi_{1}(\omega)}=\frac{1}{\phi_{1}(\omega)} \mathrm{E}\left[\exp \left\{-\omega \hat{Z}^{\left(\theta_{2}\right)}\right\}\right]-\frac{1}{\phi_{1}(\omega)} \mathrm{E}\left[\exp \left\{-\omega \hat{Z}^{\left(\theta_{1}\right)}\right\}\right]$.

Both terms are products of LSTs, corresponding to the convolution of two functions. For the first term, $\mathrm{E}\left[\exp \left\{-\omega \hat{Z}^{\left(\theta_{2}\right)}\right\}\right] / \phi_{1}(\omega)$, observe that $\mathrm{E}\left[\exp \left\{-\omega \hat{Z}^{\left(\theta_{2}\right)}\right\}\right]$ is the LST of a function with mass on $(K, \infty)$. Hence, the inverse of the first term has no mass on $(0, K]$. The inverse of the second term equals $\int_{0}^{x} W_{1}(y) \mathrm{d} y * W_{2}^{(T)}(x ; K)$. Summarizing, we obtain, for $x \in(0, K]$,

$$
\mathrm{P}(Z<x)=\frac{\hat{P}_{\downarrow K}}{\mathrm{E}\left[\theta_{2}\right]}\left(\mathrm{E}\left[\hat{L}_{2}^{\left(\theta_{2}-\theta_{1}\right)}\right] W_{1}(x)+\frac{1}{\xi} W_{2}^{(T)}(x ; K)-\int_{0}^{x} W_{2}^{(T)}(x-y ; K) W_{1}(y) \mathrm{d} y\right) .
$$

Note that the distribution of $Z$ on $(0, K]$ again depends only on $\mathrm{P}(Z<x)$ for $x \in(K, \infty)$ through a constant.

Step 3: Workload distribution on $(K, \infty)$. Using (5.3), we have completely determined the LST of $Z$. In this step we apply Laplace inversion similar to step 2 to obtain its distribution on $(K, \infty)$.

In particular, assume that the process is in steady state and consider the workload embedded at epochs when the Poisson observer finds a workload smaller than $K$. Again, to describe the workload process between these embedded epochs, we introduce the following regenerative alternating Lévy process $\left\{\tilde{Z}^{(t)}, t \geq 0\right\}$. Let $\tilde{Z}^{(0)}$ be determined according to the conditional distribution $\mathrm{P}(Z<x \mid Z<K)$ for $x \in(0, K)$, independent of the past evolution, and define $\theta_{1}=T$. During this first period the Lévy exponent is taken to be $\phi_{1}(\cdot)$. At time $\theta_{1}$, the Lévy exponent is changed into $\phi_{2}(\cdot)$. We define $\tilde{\theta}_{2}>0$ as the first observer instant with a workload smaller than $K$. As in step $0, \tilde{\theta}_{2}$ may coincide with $\theta_{1}$. Because of the stationarity of the embedded process, we have $\mathrm{E}\left[\exp \left\{-\omega \tilde{Z}^{(0)}\right\}\right]=\mathrm{E}\left[\exp \left\{-\omega \tilde{Z}^{\left(\tilde{\theta}_{2}\right)}\right\}\right]$. Lemma A.1 then reads

$$
\mathrm{E}\left[\mathrm{e}^{-\omega \tilde{Z}}\right]=\frac{\mathrm{E}\left[\tilde{L}_{1}^{\left(\theta_{1}\right)}\right]+\mathrm{E}\left[\tilde{L}_{2}^{\left(\tilde{\theta}_{2}-\theta_{1}\right)}\right]}{\mathrm{E}\left[\tilde{\theta}_{2}\right]} \frac{\omega}{\phi_{2}(\omega)}+\frac{\phi_{2}(\omega)-\phi_{1}(\omega)}{\phi_{2}(\omega)} \frac{1}{\mathrm{E}\left[\tilde{\theta}_{2}\right]} \mathrm{E}\left[\int_{s=0}^{\theta_{1}} \exp \left\{-\omega \tilde{Z}^{(s)}\right\} \mathrm{d} s\right] .
$$


It follows from similar arguments as in step 2 that the steady-state distribution of the actual process $Z$ and of the regenerative process $\tilde{Z}$ are identical. Using the Palm inversion formula [3], [33], it readily follows that the time stationary LST equals $\zeta(\omega)$ :

$$
\zeta(\omega)=\frac{1}{\mathrm{E}\left[\tilde{\theta}_{2}\right]} \mathrm{E}\left[\int_{0}^{\tilde{\theta}_{2}} \exp \left\{-\omega Z^{(s)}\right\} \mathrm{d} s\right]=\frac{1}{\mathrm{E}\left[\tilde{\theta}_{2}\right]} \mathrm{E}\left[\int_{0}^{\tilde{\theta}_{2}} \exp \left\{-\omega \tilde{Z}^{(s)}\right\} \mathrm{d} s\right]=\mathrm{E}\left[\mathrm{e}^{-\omega \tilde{Z}}\right],
$$

where the second step is by construction of $\tilde{Z}$ and the third step is by regeneration theory.

Remark 6.3. Using similar arguments as in Remark 6.1, it follows that (5.3) and (6.7) are identical. Specifically, for $x \in(0, K]$, writing $\mathrm{P}(Z<x)=\mathrm{P}(Z<x \mid Z<K) \mathrm{P}(Z<K)$, we have

$$
\begin{aligned}
\mathrm{E}\left[\int_{0}^{\theta_{1}} \exp \left\{-\omega \tilde{Z}^{(s)}\right\} \mathrm{d} s\right] & =\int_{0}^{K} \mathrm{E}_{x}\left[\int_{0}^{\theta_{1}} \exp \left\{-\omega \tilde{Z}^{(s)}\right\} \mathrm{d} s\right] \mathrm{dP}(Z<x \mid Z<K) \\
& =\frac{\mathrm{E}\left[\theta_{1}\right]}{\mathrm{P}(Z<K)} \int_{0}^{K} \mathrm{E}_{x}\left[\exp \left\{-\omega Z_{1}^{(T)}\right\}\right] \mathrm{dP}(Z<x),
\end{aligned}
$$

where the second step follows from (2.4). The fraction of time that the Lévy exponent is $\phi_{1}(\cdot)$ equals the fraction of observers finding a workload smaller than $K$, and, hence, using the PASTA property again, also equals the fraction of time that the workload is smaller than $K$. An application of the Palm inversion formula then provides $\mathrm{P}(Z<K)=\mathrm{E}\left[\theta_{1}\right] / \mathrm{E}\left[\tilde{\theta}_{2}\right]$. Using Theorem 2.2 and combining the above, we deduce that the second terms on the right-hand sides of (5.3) and (6.7) are identical, and (6.7) may thus be presented in the form of (3.3).

For the first term on the right-hand side of (6.7), we have

$$
\frac{\mathrm{E}\left[\tilde{L}_{2}^{\left(\tilde{\theta}_{2}-\theta_{1}\right)}\right]+\mathrm{E}\left[\tilde{L}_{1}^{\left(\theta_{1}\right)}\right]}{\mathrm{E}\left[\tilde{\theta}_{2}\right]}=\lim _{t \rightarrow \infty} \frac{\mathrm{E}\left[L^{(t)}\right]}{t}=Q,
$$

where the first step is an application of the Palm inversion formula and the second step follows from starting in stationarity.

It thus remains to find the time stationary distribution of $\tilde{Z}$. Using (2.4) and Theorem 2.2, it is easy to obtain the distribution on the first interval of the alternating process. Using Lemma A.2, we have

$$
\begin{aligned}
\mathrm{E}\left[\mathrm{e}^{-\omega \tilde{Z}}\right]= & \frac{\mathrm{E}\left[\tilde{L}_{2}^{\left(\tilde{\theta}_{2}-\theta_{1}\right)}\right]}{\mathrm{E}\left[\tilde{\theta}_{2}\right]} \frac{\omega}{\phi_{2}(\omega)}+\frac{1}{\mathrm{E}\left[\tilde{\theta}_{2}\right]} \frac{\mathrm{E}\left[\exp \left\{-\omega \tilde{Z}^{\left(\tilde{\theta}_{2}\right)}\right\}\right]-\mathrm{E}\left[\exp \left\{-\omega \tilde{Z}^{\left(\theta_{1}\right)}\right\}\right]}{\phi_{2}(\omega)} \\
& +\frac{1}{\mathrm{E}\left[\tilde{\theta}_{2}\right]} \mathrm{E}\left[\int_{s=0}^{\theta_{1}} \exp \left\{-\omega \tilde{Z}^{(s)}\right\} \mathrm{d} s\right] .
\end{aligned}
$$

From Theorem 2.1, the Laplace inverse of the first term is readily obtained, giving $W_{2}(\cdot)$ times a constant. Applying (2.4) and Theorem 2.2, we also directly obtain the inverse of the third term as $\mathrm{E}\left[\theta_{1}\right] / \mathrm{E}\left[\tilde{\theta}_{2}\right]$ times

$$
\tilde{W}_{\text {cond }}^{(T)}(x):=\int_{0}^{K} W_{1}^{(T)}(x ; y) \mathrm{dP}(Z<y \mid Z<K)=\frac{1}{\mathrm{P}(Z<K)} \int_{0}^{K} W_{1}^{(T)}(x ; y) \mathrm{dP}(Z<y) .
$$


Since we have determined the distribution of $Z$ on $[0, K]$, we have also found $\tilde{W}_{\text {cond }}^{(T)}(\cdot)$ (as mentioned in Section 5, we note that its precise form may be rather involved). Finally, rewrite the second term of (6.8) as

$$
\begin{aligned}
& \frac{\mathrm{E}\left[\exp \left\{-\omega \tilde{Z}^{\left(\tilde{\theta}_{2}\right)}\right\}\right]-\mathrm{E}\left[\exp \left\{-\omega \tilde{Z}^{\left(\theta_{1}\right)}\right\}\right]}{\phi_{2}(\omega)} \\
& \quad=\frac{\omega}{\phi_{2}(\omega)}\left(\frac{1-\mathrm{E}\left[\exp \left\{-\omega \tilde{Z}^{\left(\theta_{1}\right)}\right\}\right]}{\omega}-\frac{1-\mathrm{E}\left[\exp \left\{-\omega \tilde{Z}^{\left(\tilde{\theta}_{2}\right)}\right\}\right]}{\omega}\right) .
\end{aligned}
$$

Using Theorem 2.1 and Definition 2.1, it follows directly that $\omega / \phi_{2}(\omega)$ is the LST of $W_{2}(\cdot)$. Observing that we again have the difference of two convolutions, we may apply Laplace inversion to both terms separately. Note that $\left(1-\mathrm{E}\left[\exp \left\{-\omega \tilde{Z}^{\left(\theta_{1}\right)}\right\}\right]\right) / \omega$ is the LST of $\int_{0}^{x}\left(1-\tilde{W}_{\text {cond }}^{(T)}(y)\right) \mathrm{d} y$. Equivalently, $\left(1-\mathrm{E}\left[\exp \left\{-\omega \tilde{Z}^{\left(\tilde{\theta}_{2}\right)}\right\}\right]\right) / \omega$ is the LST of $\int_{0}^{x}(1-\mathrm{P}(Z<$ $y \mid Z<K)) \mathrm{d} y$ with $x \in(0, K]$. In fact, both terms correspond to the integrated tail distribution. Using the fact that a product of LSTs corresponds to a convolution of two functions, it is now easy to apply Laplace inversion to this second term.

Summarizing, we have, for $x \in(K, \infty)$,

$$
\begin{aligned}
\mathrm{P}(Z<x)=\frac{1}{\mathrm{E}\left[\tilde{\theta}_{2}\right]}\left(\mathrm{E}\left[\tilde{L}_{2}^{\left(\tilde{\theta}_{2}-\theta_{1}\right)}\right] W_{2}(x)+\int_{0}^{x} W_{2}(x-y)\left(1-\tilde{W}_{\text {cond }}^{(T)}(y)\right) \mathrm{d} y\right. \\
\\
\left.\quad-\int_{0}^{K} W_{2}(x-y)(1-\mathrm{P}(Z<y \mid Z<K)) \mathrm{d} y+\frac{1}{\xi} \tilde{W}_{\text {cond }}^{(T)}(x)\right) .
\end{aligned}
$$

Remark 6.4. Similar to Remark 6.2, we may give a different representation for the second term on the right-hand side of (6.8). In particular, for this second term, we may write $\frac{\mathrm{E}\left[\exp \left\{-\omega \tilde{Z}^{\left(\tilde{\theta}_{2}\right)}\right\}\right]-\mathrm{E}\left[\exp \left\{-\omega \tilde{Z}^{\left(\theta_{1}\right)}\right\}\right]}{\phi_{2}(\omega)}=\frac{1}{\phi_{2}(\omega)} \mathrm{E}\left[\exp \left\{-\omega \tilde{Z}^{\left(\tilde{\theta}_{2}\right)}\right\}\right]-\frac{1}{\phi_{2}(\omega)} \mathrm{E}\left[\exp \left\{-\omega \tilde{Z}^{\left(\theta_{1}\right)}\right\}\right]$.

Using Theorem 2.1 and Definition 2.1, we easily find that $1 / \phi_{2}(\omega)$ is the LST of $\int_{0}^{x} W_{2}(y) \mathrm{d} y$. Observing that we again have the sum of two convolution terms, we may easily apply Laplace inversion to both terms separately. In particular, for the first term, $E\left[\exp \left\{-\omega \tilde{Z}^{\left(\theta_{2}\right)}\right\}\right] / \phi_{2}(\omega)$, we obtain an incomplete convolution between $\int_{0}^{x} W_{2}(y) \mathrm{d} y$ and $\mathrm{P}(Z<x \mid Z<K)$ (see also below). For the second term, $\mathrm{E}\left[\exp \left\{-\omega \tilde{Z}^{\left(\theta_{1}\right)}\right\}\right] / \phi_{2}(\omega)$, we have a convolution of $\int_{0}^{x} W_{2}(y) \mathrm{d} y$ with $\tilde{W}_{\text {cond }}^{(T)}(\cdot)$. Summarizing, we obtain, for $x \in(K, \infty)$,

$$
\begin{gathered}
\mathrm{P}(Z<x)=\frac{1}{\mathrm{E}\left[\tilde{\theta}_{2}\right]}\left(\mathrm{E}\left[\tilde{L}_{2}^{\left(\tilde{\theta}_{2}-\theta_{1}\right)}\right] W_{2}(x)+\int_{0}^{K} \int_{0}^{x-y} W_{2}(z) \mathrm{d} z \mathrm{dP}(Z<y \mid Z<K)\right. \\
\left.-\int_{0}^{x} \tilde{W}_{\text {cond }}^{(T)}(x-y) W_{2}(y) \mathrm{d} y+\frac{1}{\xi} \tilde{W}_{\text {cond }}^{(T)}(x)\right) .
\end{gathered}
$$

Alternatively, conditioning on $\int_{0}^{x} W_{2}(y) \mathrm{d} y$ for the second convolution, we may represent the distribution of $Z$ on $(K, \infty)$ as

$$
\begin{gathered}
\mathrm{P}(Z<x)=\frac{1}{\mathrm{E}\left[\tilde{\theta}_{2}\right]}\left(\mathrm{E}\left[\tilde{L}_{2}^{\left(\tilde{\theta}_{2}-\theta_{1}\right)}\right] W_{2}(x)+\int_{x-K}^{x} \mathrm{P}(Z<x-y \mid Z<K) W_{2}(y) \mathrm{d} y\right. \\
\left.-\int_{0}^{x} \tilde{W}_{\text {cond }}^{(T)}(x-y) W_{2}(y) \mathrm{d} y+\frac{1}{\xi} \tilde{W}_{\text {cond }}^{(T)}(x)\right) .
\end{gathered}
$$


Step 4: Determination of the constants. It follows from Remarks 6.1 and 6.3 that

$$
\frac{\mathrm{E}\left[\hat{L}_{2}^{\left(\theta_{2}-\theta_{1}\right)}\right]+\mathrm{E}\left[\hat{L}_{1}^{\left(\theta_{1}\right)}\right]}{\mathrm{E}\left[\theta_{2}\right]}=\frac{\mathrm{E}\left[\tilde{L}_{2}^{\left(\tilde{\theta}_{2}-\theta_{1}\right)}\right]+\mathrm{E}\left[\tilde{L}_{1}^{\left(\theta_{1}\right)}\right]}{\mathrm{E}\left[\tilde{\theta}_{2}\right]}=Q .
$$

Using (2.4) and the final observation in Remark 2.1, it can be easily verified (see also [10, Equation (3.6)]) that

$$
\frac{\mathrm{E}\left[\tilde{L}_{1}^{\left(\theta_{1}\right)}\right]}{\mathrm{E}\left[\tilde{\theta}_{2}\right]}=\xi \int_{0}^{K} \frac{\exp \left\{-\eta_{1}(\xi) x\right\}}{\eta_{1}(\xi)} \mathrm{dP}(Z<x),
$$

which can be determined in terms of $Q$ and $\mathrm{P}(Z<K)$ by multiplying either (6.5) or (6.6) by $\exp \left\{-\eta_{1}(\xi) x\right\}$ and integrating over the interval $[0, K]$. Similarly, we have

$$
\frac{\mathrm{E}\left[\hat{L}_{1}^{\left(\theta_{1}\right)}\right]}{\mathrm{E}\left[\theta_{2}\right]}=\xi \int_{K}^{\infty} \frac{\exp \left\{-\eta_{2}(\xi) x\right\}}{\eta_{2}(\xi)} \mathrm{dP}(Z<x),
$$

which can also be expressed in terms of $Q$ and $\mathrm{P}(Z<K)$ by combining the above with the definition of $Q$, i.e. (5.1). From the observations in Remarks 6.1 and 6.3, we also have $1 / \mathrm{E}\left[\theta_{2}\right]=\xi \mathrm{P}(Z \geq K)$ and $1 / \mathrm{E}\left[\tilde{\theta}_{2}\right]=\xi \mathrm{P}(Z<K)$. It remains to determine $Q$ and $\mathrm{P}(Z<K)$. Letting $\omega \downarrow 0$ in $(6.1)$, we obtain

$$
Q=\phi_{2}^{\prime}(0)-\left(\phi_{2}^{\prime}(0)-\phi_{1}^{\prime}(0)\right) \mathrm{P}(Z<K) .
$$

Note that letting $\omega \downarrow 0$ in (6.7) gives the same equation (this also provides the first equality in (6.9)). In addition, $\mathrm{P}(Z<K)$ can be directly obtained by substituting $x=K$ into either (6.5) or (6.6), with $\hat{P}_{\downarrow K}$ given by (6.3). These latter two equations determine the constants $Q$ and $\mathrm{P}(Z<K)$ and, thus, the remaining constants.

\section{Appendix A. Alternating Lévy processes}

Here, we analyze alternating Lévy processes without negative jumps. Consider a regeneration cycle, and let some (possibly random) level $Z^{(0)} \geq 0$ be the starting level of the cycle. At these regeneration points a first period starts, consisting of a reflected Lévy process without negative jumps and a Lévy exponent $\phi_{I}(\cdot)$. At some stopping time $\tau_{I}$ the first interval ends, and the Lévy exponent is changed into $\phi_{I I}(\cdot)$ until the end of the regeneration cycle, denoted by time $\tau_{I I}$. Denote the starting time of interval $i=I, I I$ by $\tau_{i}^{s}$, i.e. $\tau_{I}^{s}=0$ and $\tau_{I I}^{s}=\tau_{I}$. For convenience, the first period is referred to as interval $I$ and the second period as interval $I I_{\tau^{s}}$ The reflected process is denoted by $\left\{Z^{(t)}, t \geq 0\right\}$ and is defined as $Z^{(t)}=Z^{\left(\tau_{i}^{s}\right)}+X_{i}^{\left(t-\tau_{i}^{s}\right)}+L_{i}^{\left(t-\tau_{i}^{s}\right) \mid z}$ for $t \in\left[\tau_{i}^{s}, \tau_{i}\right), i=I, I I$, where we use the notation $z=Z^{(0)}$. Here, $L_{i}^{(t) \mid z}=-\inf _{0 \leq u \leq t}\left[Z^{\left(\tau_{i}^{s}\right)}+X_{i}^{(u)}\right]^{-}$(in this appendix we explicitly indicate the dependence of $L$ on the initial value $Z^{(0)}=z$, but, for ease of presentation, this is often omitted in the remainder of the paper). Denote the steady-state version of $\left\{Z^{(t)}, t \geq 0\right\}$, assuming that it exists, by $Z$.

We note that the model introduced above is not very natural in its full generality. However, various natural models might be considered as a special case. The most prominent model is the M/G/1 dam (see Section 3); define $Z^{(0)}=K, \tau_{I}$ as the first upcrossing of $K$, and $\tau_{I I}$ as the subsequent downcrossing of $K$. The equations in Lemmas A.1 and A.2, below, appear in various parts of the paper. 
Lemma A.1. For an alternating Lévy process as described above, the LST of the steady-state workload satisfies the following equations:

$$
\begin{aligned}
\mathrm{E}\left[\mathrm{e}^{-\omega Z}\right]= & \frac{\mathrm{E}\left[L_{I I}^{\left(\tau_{I I}-\tau_{I}\right) \mid z}\right]+\mathrm{E}\left[L_{I}^{\left(\tau_{I}\right) \mid z}\right]}{\mathrm{E}\left[\tau_{I I}\right]} \frac{\omega}{\phi_{I I}(\omega)}+\frac{\mathrm{E}\left[\exp \left\{-\omega Z^{\left(\tau_{I I}\right)}\right\}\right]-\mathrm{E}\left[\exp \left\{-\omega Z^{(0)}\right\}\right]}{\phi_{I I}(\omega) \mathrm{E}\left[\tau_{I I}\right]} \\
& +\frac{\phi_{I I}(\omega)-\phi_{I}(\omega)}{\phi_{I I}(\omega)} \frac{1}{\mathrm{E}\left[\tau_{I I}\right]} \mathrm{E}\left[\int_{s=0}^{\tau_{I}} \exp \left\{-\omega Z^{(s)}\right\} \mathrm{d} s\right] .
\end{aligned}
$$

Also,

$$
\begin{aligned}
\mathrm{E}\left[\mathrm{e}^{-\omega Z}\right]= & \frac{\mathrm{E}\left[L_{I I}^{\left(\tau_{I I}-\tau_{I}\right) \mid z}\right]+\mathrm{E}\left[L_{I}^{\left(\tau_{I}\right) \mid z}\right]}{\mathrm{E}\left[\tau_{I I}\right]} \frac{\omega}{\phi_{I}(\omega)}+\frac{\mathrm{E}\left[\exp \left\{-\omega Z^{\left(\tau_{I I}\right)}\right\}\right]-\mathrm{E}\left[\exp \left\{-\omega Z^{(0)}\right\}\right]}{\phi_{I}(\omega) \mathrm{E}\left[\tau_{I I}\right]} \\
& +\frac{\phi_{I}(\omega)-\phi_{I I}(\omega)}{\phi_{I}(\omega)} \frac{1}{\mathrm{E}\left[\tau_{I I}\right]} \mathrm{E}\left[\int_{s=\tau_{I}}^{\tau_{I I}} \exp \left\{-\omega Z^{(s)}\right\} \mathrm{d} s\right] .
\end{aligned}
$$

Proof. To derive the steady-state distribution of this process, we use the following martingale [1], [22]: for $i=I, I I$,

$$
M_{i}^{(t)}=\phi_{i}(\omega) \int_{s=0}^{t} \exp \left\{-\omega Z^{(s)}\right\} \mathrm{d} s-\exp \left\{-\omega Z^{(t)}\right\}+\exp \left\{-\omega Z^{(0)}\right\}-\omega L_{i}^{(t) \mid z} .
$$

Application of the optional sampling theorem, with stopping time $\tau_{I}$, to this martingale (with $i=I$ ) yields (cf. [1], [22])

$$
\phi_{I}(\omega) \mathrm{E}\left[\int_{s=0}^{\tau_{I}} \exp \left\{-\omega Z^{(s)}\right\} \mathrm{d} s\right]=\mathrm{E}\left[\exp \left\{-\omega Z^{\left(\tau_{I}\right)}\right\}\right]-\mathrm{E}\left[\exp \left\{-\omega Z^{(0)}\right\}\right]+\omega \mathrm{E}\left[L_{I}^{\left(\tau_{I}\right) \mid z}\right] .
$$

Note that the end point of this first period, i.e. $Z^{\left(\tau_{I}\right)}$, is also the starting point of the second interval. Rewriting the above, we have

$$
\mathrm{E}\left[\exp \left\{-\omega Z^{\left(\tau_{I}\right)}\right\}\right]=\phi_{I}(\omega) \mathrm{E}\left[\int_{s=0}^{\tau_{I}} \exp \left\{-\omega Z^{(s)}\right\} \mathrm{d} s\right]+\mathrm{E}\left[\exp \left\{-\omega Z^{(0)}\right\}\right]-\omega \mathrm{E}\left[L_{I}^{\left(\tau_{I}\right) \mid z}\right]
$$

For the second interval, we apply the optional sampling theorem, with stopping time $\tau_{I I}$, to this martingale (starting at $\tau_{I}$ instead of at 0 ), yielding

$$
\begin{aligned}
\phi_{I I}(\omega) \mathrm{E}\left[\int_{s=\tau_{I}}^{\tau_{I I}} \exp \left\{-\omega Z^{(s)}\right\} \mathrm{d} s\right] \\
=\mathrm{E}\left[\exp \left\{-\omega Z^{\left(\tau_{I I}\right)}\right\}\right]-\mathrm{E}\left[\exp \left\{-\omega Z^{\left(\tau_{I}\right)}\right\}\right]+\omega \mathrm{E}\left[L_{I I}^{\left(\tau_{I I}-\tau_{I}\right) \mid z}\right] \\
=-\phi_{I}(\omega) \mathrm{E}\left[\int_{s=0}^{\tau_{I}} \exp \left\{-\omega Z^{(s)}\right\} \mathrm{d} s\right]+\mathrm{E}\left[\exp \left\{-\omega Z^{\left(\tau_{I I}\right)}\right\}\right]-\mathrm{E}\left[\exp \left\{-\omega Z^{(0)}\right\}\right] \\
\\
+\omega \mathrm{E}\left[L_{I I}^{\left(\tau_{I I}-\tau_{I}\right) \mid z}\right]+\omega \mathrm{E}\left[L_{I}^{\left(\tau_{I}\right) \mid z}\right]
\end{aligned}
$$

where the second step follows from (A.3). From regeneration theory, it follows that the LST of the steady-state workload $Z$ in a queue with alternating exponents is given by

$$
\mathrm{E}\left[\mathrm{e}^{-\omega Z}\right]=\frac{1}{\mathrm{E}\left[\tau_{I I}\right]}\left(\mathrm{E}\left[\int_{s=0}^{\tau_{I}} \exp \left\{-\omega Z^{(s)}\right\} \mathrm{d} s\right]+\mathrm{E}\left[\int_{s=\tau_{I}}^{\tau_{I I}} \exp \left\{-\omega Z^{(s)}\right\} \mathrm{d} s\right]\right) .
$$


Hence, dividing (A.5) by $\phi_{I I}(\omega)$, adding $\mathrm{E}\left[\int_{s=0}^{\tau_{I}} \exp \left\{-\omega Z^{(s)}\right\} \mathrm{d} s\right]$ to both sides, and then dividing by $\mathrm{E}\left[\tau_{I I}\right]$, we obtain

$$
\begin{aligned}
\mathrm{E}\left[\mathrm{e}^{-\omega Z}\right]= & \frac{\mathrm{E}\left[L_{I I}^{\left(\tau_{I I}-\tau_{I}\right) \mid z}\right]+\mathrm{E}\left[L_{I}^{\left(\tau_{I}\right) \mid z}\right]}{\mathrm{E}\left[\tau_{I I}\right]} \frac{\omega}{\phi_{I I}(\omega)}+\frac{\mathrm{E}\left[\exp \left\{-\omega Z^{\left(\tau_{I I}\right)}\right\}\right]-\mathrm{E}\left[\exp \left\{-\omega Z^{(0)}\right\}\right]}{\phi_{I I}(\omega) \mathrm{E}\left[\tau_{I I}\right]} \\
& +\frac{1}{\mathrm{E}\left[\tau_{I I}\right]} \frac{\phi_{I I}(\omega)-\phi_{I}(\omega)}{\phi_{I I}(\omega)} \mathrm{E}\left[\int_{s=0}^{\tau_{I}} \exp \left\{-\omega Z^{(s)}\right\} \mathrm{d} s\right] .
\end{aligned}
$$

Hence, we have derived (A.1). Using (A.6) to rewrite the final term in (A.7) and some rewriting provides (A.2).

In addition to the M/G/1 dam, the equations of Lemma A.1 can be useful in several special cases, especially when $Z^{(0)}=Z^{\left(\tau_{I I}\right)}$ and $\phi_{I}$ and $\phi_{I I}$ are related. Typical examples are Lévy storage models where the output is shut off every time the system reaches 0 (as in, for instance, vacation models or service according to $D$-policies); see also [21]. As an easy application, we next consider an M/G/1 queue with service rate $r$ and an exceptional first service during a busy period. Moreover, the result is a slight extension of familiar results, where service at unit speed is assumed; see, e.g. [35, p. 128] or [36],

Example A.1. In this example we consider an M/G/1 queue with arrival rate $\lambda$, service speed $r$, and generic service requirement $B_{I I}$. Each first customer in a busy period receives an exceptional service that is generically denoted by $B_{I}$. Let $\beta_{i}(\cdot), i=I, I I$, be the LST of $B_{i}$ and denote by $\beta_{i}$ its mean.

This model can easily be analyzed using Lemma A.1 and some trivial observations. In the general model with alternating exponents, let $Z^{(0)}=0$, define $\tau_{I}$ as the first customer arrival epoch, and let $\tau_{I I}:=\inf \left[t>\tau_{I}: Z^{(t)}=0\right]$ be the end of the busy cycle. We may then take $\phi_{i}(\omega)=r \omega-\lambda+\lambda \beta_{i}(\omega), i=I, I I$. Note that there is no reflection in the second interval; hence, $\mathrm{E}\left[L_{I I}^{\left(\tau_{I I}-\tau_{I}\right) \mid 0}\right]=0$. By definition of $\tau_{I}$ we have for the first interval that $Z^{(s)}=0$, with $s \in\left[0, \tau_{I}\right)$, implying that $\mathrm{E}\left[\int_{0}^{\tau_{I}} \exp \left\{-\omega Z^{(s)}\right\} \mathrm{d} s\right]=\mathrm{E}\left[\tau_{I}\right]$. Moreover, since it holds for the free process that $X^{\left(\tau_{I}^{-}\right)}=-r \tau_{I}$, we have $\mathrm{E}\left[L_{I}^{\left(\tau_{I}\right) \mid 0}\right]=r \mathrm{E}\left[\tau_{I}\right]$. Substituting the above into (A.1) and some straightforward rewriting yields

$$
\mathrm{E}\left[\mathrm{e}^{-\omega Z}\right]=\frac{\mathrm{E}\left[\tau_{I}\right]}{\mathrm{E}\left[\tau_{I I}\right]} \frac{r \omega-\lambda \beta_{I}(\omega)+\lambda \beta_{I I}(\omega)}{r \omega-\lambda+\lambda \beta_{I I}(\omega)} .
$$

The constant $\mathrm{E}\left[\tau_{I}\right] / \mathrm{E}\left[\tau_{I I}\right]$ can be obtained by letting $\omega \downarrow 0$ and applying l'Hôpital's rule, giving the final result

$$
\mathrm{E}\left[\mathrm{e}^{-\omega Z}\right]=\frac{1-\rho}{1+\lambda \beta_{I} / r-\rho} \frac{r \omega-\lambda \beta_{I}(\omega)+\lambda \beta_{I I}(\omega)}{r \omega-\lambda+\lambda \beta_{I I}(\omega)},
$$

where $\rho=\lambda \beta_{I I} / r$. We refer the reader to, for example, [35, p. 128] or [36] for the case $r \equiv 1$.

Another equation that is useful when $\mathrm{E}\left[\exp \left\{-\omega Z^{\left(\tau_{I}\right)}\right\}\right]$ and the steady-state workload distribution of the first interval can be determined separately is presented below. It follows by dividing both sides of (A.4) by $\phi_{I I}(\omega)$ and adding $\mathrm{E}\left[\int_{s=0}^{\tau_{I}} \exp \left\{-\omega Z^{(s)}\right\} \mathrm{d} s\right]$ to both sides. 
Lemma A.2. For an alternating Lévy process as described above, we have the following relations:

$$
\begin{aligned}
\mathrm{E}\left[\int_{s=0}^{\tau_{I I}} \exp \left\{-\omega Z^{(s)}\right\} \mathrm{d} s\right]= & \frac{\mathrm{E}\left[L_{I I}^{\left(\tau_{I I}-\tau_{I}\right) \mid z}\right] \omega}{\phi_{I I}(\omega)}+\frac{\mathrm{E}\left[\exp \left\{-\omega Z^{\left(\tau_{I I}\right)}\right\}\right]-\mathrm{E}\left[\exp \left\{-\omega Z^{\left(\tau_{I}\right)}\right\}\right]}{\phi_{I I}(\omega)} \\
& +\mathrm{E}\left[\int_{s=0}^{\tau_{I}} \exp \left\{-\omega Z^{(s)}\right\} \mathrm{d} s\right] .
\end{aligned}
$$

\section{Acknowledgements}

This research was done within the framework of the BRICKS project and the European Network of Excellence Euro-NGI. Part of this research was done while the first author was affiliated to CWI, Amsterdam, The Netherlands.

\section{References}

[1] Asmussen, S. (2003). Applied Probability and Queues, 2nd edn. Springer, New York.

[2] Avram, F., Kyprianou, A. E. And Pistorius, M. R. (2004). Exit problems for spectrally negative Lévy processes and applications to (Canadized) Russian options. Ann. Appl. Prob. 14, 215-238.

[3] Baccelli, F. and Brémaud, P. (2003). Elements of Queueing Theory, 2nd edn. Springer, Berlin.

[4] BekKer, R. And Boxma, O. J. (2007). An M/G/1 queue with adaptable service speed. Stoch. Models 23, 373-396.

[5] Bekker, R., Boxma, O. J. And Resing, J. A. C. (2008). Queues with service speed adaptations. Statist. Neerlandica 62, 441-457.

[6] Bertoin, J. (1996). Lévy Processes (Camb. Tracts Math. 121). Cambridge University Press.

[7] Bertoin, J. (1997). Exponential decay and ergodicity of completely asymmetric Lévy processes in a finite interval. Ann. Appl. Prob. 7, 156-169.

[8] Bingham, N. H. (1975). Fluctuation theory in continuous time. Adv. Appl. Prob. 7, 705-766.

[9] Boxma, O. J. And Lotov, V. I. (1996). On a class of one-dimensional random walks. Markov Process. Relat. Fields 2, 349-362.

[10] Boxma, O. J., Perry, D. And Stadje, W. (2001). Clearing models for M/G/1 queues. Queueing Systems 38, 287-306.

[11] Brockwell, P. J., Resnick, S. I. And Tweedie, R. L. (1982). Storage processes with general release rule and additive inputs. Adv. Appl. Prob. 14, 392-433.

[12] Cohen, J. W. (1976). On Regenerative Processes in Queueing Theory (Lecture Notes Econom. Math. Systems 121). Springer, Berlin.

[13] Cohen, J. W. (1976). On the optimal switching level for an M/G/1 queueing system. Stoch. Process. Appl. 4, 297-316.

[14] Cohen, J. W. (1982). The Single Server Queue, 2nd edn. North-Holland, Amsterdam.

[15] Cohen, J. W. And Rubinovitch, M. (1977). On level crossings and cycles in dam processes. Math. Operat. Res. 2, 297-310.

[16] Dshalalow, J. H. (1997). Queueing systems with state dependent parameters. In Frontiers in Queueing, ed. J. H. Dshalalow, CRC Press, Boca Raton, FL, pp. 61-116.

[17] Gaver, D. P. and Miller, R. G. (1962). Limiting distributions for some storage problems. In Studies in Applied Probability and Management Science, Stanford University Press, pp. 110-126.

[18] Gerber, H. U. and ShiU, E. S. W. (2006). On optimal dividends: from reflection to refraction. J. Comput. Appl. Math. 186, 4-22.

[19] Gerber, H. U. and Shiu, E. S. W. (2006). On optimal dividend strategies in the compound Poisson model. N. Amer. Actuarial J. 10, 76-93.

[20] Hubalek, F. and Kyprianou, A. E. (2007). Old and new examples of scale functions for spectrally negative Lévy processes. Preprint. Available at http://arxiv.org/abs/0801.0393v2.

[21] Kella, O. (1998). An exhaustive Lévy storage process with intermittent output. Stoch. Models 14, 979-992.

[22] Kella, O. and Whitt, W. (1992). Useful martingales for stochastic storage processes with Lévy input. J. Appl. Prob. 29, 396-403.

[23] Kyprianou, A. E. and Loeffen, R. L. (2008). Refracted Lévy processes. To appear in Ann. Inst. H. Poincaré. Preprint available at http://arxiv.org/abs/0801.4655.

[24] Kyprianou, A. E. AND Palmowski, Z. (2005). A martingale review of some fluctuation theory for spectrally negative Lévy processes. In Séminaire de Probabilités XXXVIII (Lecture Notes Math. 1857), Springer, Berlin, pp. 16-29. 
[25] Kyprianou, A. E. And Rivero, V. (2008). Special, conjugate and complete scale functions for spectrally negative Lévy processes. Electron. J. Prob. 13, 1672-1701.

[26] Lin, X. S. and Pavlova, K. P. (2006). The compound Poisson risk model with a threshold dividend strategy. Insurance Math. Econom. 38, 57-80.

[27] NGUYEn-Ngoc, L. AND Yor, M. (2005). Some martingales associated to reflected Lévy processes. In Séminaire de Probabilités XXXVIII (Lecture Notes Math. 1857), Springer, Berlin, pp. 42-69.

[28] Pistorius, M. R. (2003). On doubly reflected completely asymmetric Lévy processes. Stoch. Process. Appl. 107, 131-143.

[29] Prabhu, N. U. (1965). Queues and Inventories. John Wiley, New York.

[30] Prabhu, N. U. (1980). Stochastic Storage Processes (Appl. Math. 15). Springer, New York.

[31] Roughan, M. and Pearce, C. E. M. (2002). Martingale methods for analysing single-server queues. Queueing Systems 41, 205-239.

[32] Rubinovitch, M. and Cohen, J. W. (1980). Level crossings and stationary distributions for general dams. J. Appl. Prob. 17, 218-226.

[33] Sigman, K. (1995). Stationary Marked Point Processes. Chapman and Hall, New York.

[34] Surya, B. A. (2008). Evaluating scale functions of spectrally negative Lévy processes. J. Appl. Prob. 45, 135149.

[35] Takagi, H. (1991). Queueing Analysis: A Foundation of Performance Evaluation, Vol. 1. North-Holland, Amsterdam.

[36] Welch, P. D. (1964). On a generalized M/G/1 queuing process in which the first customer of each busy period receives exceptional service. Operat. Res. 12, 736-752. 